\title{
Symbolic Computation Applied to the Study of the Kernel of Special Classes of Paired Singular Integral Operators
}

\author{
Ana C. Conceição
}

\begin{abstract}
Operator theory has many applications in several main scientific research areas (structural mechanics, aeronautics, quantum mechanics, ecology, probability theory, electrical engineering, among others) and the importance of its study is globally acknowledged. On the study of the operator's kernel some progress has been achieved for some specific classes of singular integral operators whose properties allow the use of particular strategies. However, the existing algorithms allow, in general, to study the dimension of the kernel of some classes of singular integral operators but are not designed to be implemented on a computer. The main goal of this paper is to show how the symbolic and numeric capabilities of a computer algebra system can be used to study the kernel of special classes of paired singular integral operators with essentially bounded coefficients defined on the unit circle. It is described how some factorization algorithms can be used to compute the dimension of the kernel of special classes of singular integral operators. The analytical algorithms [ADimKerPaired-Scalar], [AKerPaired-Scalar], and [ADimKerPaired-Matrix] are presented. The design of these new algorithms was focused on the possibility of implementing on a computer all the extensive symbolic and numeric calculations present in the algorithms. For the essentially bounded hermitian coefficients case, there exist some relations with Hankel operators. The paper contains some interesting and nontrivial examples obtained with the use of a computer algebra system.
\end{abstract}

Mathematics Subject Classification (2010). Primary 68W30, 47B35, 47A68; Secondary 30E20, 47G10.

Keywords. Symbolic computation, kernel of paired singular integral operators, factorization algorithms, essentially bounded matrix functions, Wolfram Mathematica.

\section{Introduction}

The development of operator theory is motivated by the need to solve problems emerging from several fields in mathematics and physics. On the study of the operator's kernel some progress has been achieved for some classes of singular integral operators whose properties allow the use of particular strategies. However, the existing algorithms allow, in general, to study the kernel of some classes of singular integral operators but they are not designed to be implemented on a computer.

In our work we use the computer algebra system Mathematica ${ }^{1}$ to implement for the first time on a computer analytical algorithms developed by us and others within operator theory. In the last years we designed and/or implemented analytical algorithms for solving integral equations [11, 17], analytical algorithms to factorize scalar and matrix functions [16, 17], calculation techniques to compute singular integrals [15], and more recently analytical algorithms to study the spectrum [22, 23]

This research was supported by Fundação para a Ciência e Tecnologia (Portugal) through the Center for Functional Analysis, Linear Structures and Applications.

${ }^{1}$ Wolfram Mathematica is a symbolic mathematical computation program used in many scientific, engineering, and computing fields. It was conceived by Stephen Wolfram and is developed by Wolfram Research. 
and the kernel [21] of several classes of singular integral operators. It is our belief that the construction and implementation on a computer of these kind of analytical algorithms is a very interesting line of research.

Factorization theory has a long and interesting history and is closely related to the computation of singular integrals, and its roots lie in the work of Plemelj [46]. The relations between the factorization of matrix functions, Riemann-Hilbert boundary value problems, and systems of singular integral equations have been known from the early stages of factorization theory (see, for instance, [31]). At present time, the theory has wide application in the theory of linear and non-linear differential equations, in linear transport theory, in theory of diffraction of acoustic and electromagnetic waves, in theory of scattering, among others (see, for instance, [10, 18, 27, 38, 47]). Some progress has been achieved (see, for instance, $[2,9,26,28,43]$ ) for some classes of matrix functions whose properties allow the use of a particular strategy in the study of the factorization problem but there is no general method for obtaining a factorization for a given matrix function. In [16] it is described the explicit rational function factorization algorithms [ARFact-Scalar] and matrix [ARFact-Matrix] that compute explicit (left and right) factorizations of given non-singular rational matrix functions defined on the unit circle. Both algorithms were implemented using the computer algebra system Mathematica. On the determination of the partial indices, some developments have also been made but, even in the rational case (and in recent publications), the methods are difficult to apply and were not designed to be implemented on a computer (see, for instance, $[5,8,35,49]$ ). In addition, the vast majority of explicit analytical factorization methods depends on the knowledge of the zeros of scalar functions. As a consequence, in many applications in the real world, a numerical analysis of such methods is inevitable. However, due to many non-stability issues, such as the ones affecting the factorization partial indices [31], the numerical approach of factorization theory is a very difficult problem. Due to this fact, the design of new analytical methods, even if only for some restricted special classes of functions, is still very significant to the development of such theory. In particular, thanks to the symbolic computation capabilities of Mathematica, the [ARFact-Scalar] algorithm [16] always computes the factorization index of any considered factorable scalar rational function defined on the unit circle. One the other hand, the generalized factorization algorithm [AFact] uses the inner-outer factorization concept and was partially implemented on a computer using the computer algebra system Mathematica $[11,17]$. The classes of essentially bounded matrix functions that can be factorized by this analytic algorithm are closely related to the solution of the non-linear Schrödinger equation (see, for instance, [27]), to the generalized Riemann-Hilbert problem (see, for instance, [20, 21, 41, 42]), and to the study of singular integral operators related with Hankel operators [18].

On the study of the kernel of an operator the importance of the factorization theory is well known (see, for instance, [33, 34, 42]). In [20] it is described how the generalized factorization concept is related with estimates of the dimension of the kernel of some classes of singular integral operators with non-Carleman shift. In [21] it is described how the algorithms [AFact], [ARFact-Matrix], and [SInt] [15] can be used to estimate the dimension of the kernel of some classes of singular integral operators with non-Carleman shift and conjugation. In [12] it is shown how the symbolic and numeric capabilities of the computer algebra system Mathematica can be used to compute the dimension of the kernel of rational classes of paired singular integral operators.

The main goal of this paper is to show how the symbolic and numeric capabilities of a computer algebra system can be used to study the kernel of special classes of paired singular integral operators with essentially bounded coefficients defined on the unit circle. The analytical algorithms [ADimKerPaired-Scalar], [AKerPaired-Scalar], and [ADimKerPaired-Matrix] are presented. The design of these new algorithms, that use the [ARFact-Scalar] and [ARFact-Matrix] algorithms (see Figure 1 ), was focused on the possibility of implementing on a computer all the extensive symbolic and numeric calculations present in the algorithms. It is considered the one-dimensional and the matrix cases. It is also shown some relations between the dimension of the kernel of singular integral operators connected with Hankel operators and the dimension of the kernel of some special classes of 
paired singular integral operators with essentially bounded hermitian coefficients. Some interesting and nontrivial examples obtained with the use of a computer algebra system are presented.

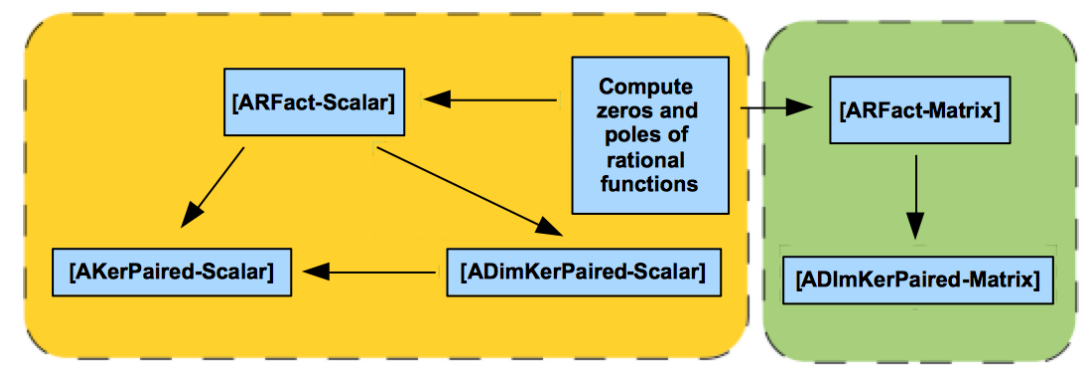

Figure 1. General flowchart.

The paper is organized as follows: Section 2 contains some basic definitions and properties of paired singular integral operators and some factorization concepts. Section 3 is dedicated to some results on the kernel of paired singular integral operators. Some relations between the generalized factorization concept and the kernel of paired singular integral operators are presented. It is considered the one-dimensional and the matrix cases. In Section 4 it is explained how the symbolic and numeric computation capabilities of a computer algebra system can be used to study the kernel of some classes of paired singular integral operators. The new algorithms [ADimKerPaired-Scalar], [AKerPaired-Scalar], and [ADimKerPaired-Matrix] are presented. Section 5 is dedicated to some special classes of paired singular integral operators with essentially bounded hermitian coefficients, defined on the unit circle.

\section{Basic concepts}

Let $\pi$ denote the unit circle ${ }^{2}$ in the complex plane. Let $\pi_{+}$and $\pi_{-}$denote the open unit disk and the exterior region of the unit circle ( $\infty$ included), respectively. As usual, $L_{\infty}(\pi)$ denotes the space of all essentially bounded functions defined on $\pi$ and $H_{\infty}(\pi)$ the class of all bounded and analytic functions in $\pi_{+}$. Let $\mathcal{R}(\pi)$ be the algebra of rational functions without poles on $\pi$ and $\mathcal{R}_{ \pm}(\pi)$ the subsets of $\mathcal{R}(\pi)$ whose elements have no poles in $\pi_{ \pm}$, respectively.

\subsection{Singular integral operators}

The study of singular integral operators has applications in different research areas, such as theory of diffraction of acoustic and electromagnetic waves, theory of scattering and of inverse scattering, and factorization theory (see, for instance, $[2,14,18,27]$ ). It is well known that the singular integral ${ }^{3}$ operator with Cauchy kernel, $S_{\pi}$, defined almost everywhere on $\pi$ by

$$
S_{\pi} \varphi(t)=\frac{1}{\pi i} \int_{\pi} \frac{\varphi(\tau)}{\tau-t} d \tau, t \in \pi,
$$

where the integral is understood in the sense of its principal value, represents a bounded linear operator in the Lebesgue space $L_{2}(\pi)$. In addition, $S_{\pi}$ is a selfadjoint and unitary operator in $L_{2}(\pi)$ (see, for instance, [34]). Thus, we can associate with $S_{\pi}$ two complementary Cauchy projection operators

$$
P_{ \pm}=\left(I \pm S_{\pi}\right) / 2 \text {, }
$$

where $I$ represents the identity operator.

\footnotetext{
${ }^{2}$ Although many of the results presented in this paper can be generalized $[7,25]$ to the space $L_{p}(\Gamma)$, where $\Gamma$ is a closed Carleson curve, we decided to state them only for $L_{2}(\pi)$ due to the use of symbolic computation for the construction of nontrivial examples.

${ }^{3}$ The [SInt] algorithm described in [15] computes (1) when the essentially bounded function $\varphi$ can be represented as

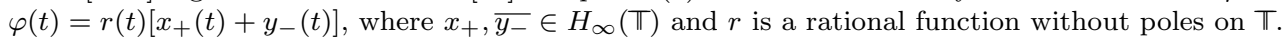


The projectors (2) allow us to decompose the space $L_{2}(\pi)$ in the topological direct sum

$$
L_{2}(\pi)=L_{2}^{+}(\pi) \oplus L_{2}^{-, 0}(\pi)
$$

where $L_{2}^{+}(\pi)=\operatorname{im} P_{+}$and $L_{2}^{-, 0}(\mathbb{\pi})=\operatorname{im} P_{-}$. We also consider the space $L_{2}^{-}(\mathbb{\pi})=L_{2}^{-, 0}(\mathbb{\pi}) \oplus \mathbb{C}$.

There exist several numerical algorithms and approximation methods for evaluating some classes of singular integrals. Also, there are several analytical techniques that allow the exact computation of singular integrals for particular cases. However, the [SInt] ${ }^{4}$ and [SIntAFact] algorithms [15] are the only analytical algorithms, up to our knowledge, written and implemented, for computing singular integrals with general essentially bounded functions defined on the unit circle. Both algorithms were implemented using the numeric and symbolic computation capabilities of Mathematica. In particular, the implementation of the [SInt] algorithm makes the results of lengthy and complex calculations available in a simple way to researchers of different areas.

Let $\varphi, \psi \in\left[L_{\infty}(\pi)\right]_{n, n}$. Operators of the form $T=\varphi I+\psi S_{\pi}$ and $\widetilde{T}=\varphi I+S_{\pi} \psi I$ are linear and bounded singular integral operators (see, for instance, [34]). In the following, these operators will be written in a more convenient form as

$$
T_{\{a, b\}}=a P_{+}+b P_{-}
$$

and

$$
\widetilde{T}_{\{a, b\}}=P_{+} a I+P_{-} b I,
$$

where $a=\varphi+\psi$ and $b=\varphi-\psi$. We will call these operators, paired singular integral operators, with coefficients $a$ and $b$.

Obviously, since $S_{\pi}=P_{+}-P_{-}, S_{\pi}$ is a paired singular integral operator that belongs to classes (3) and (4).

\subsection{Factorization concepts}

Let us now introduce some factorization concepts (see, for instance, [10, 30, 42, 45, 48]) that are related to the study of the kernel of some special classes of paired singular integral operators.

\subsubsection{Generalized factorization.}

Let us now introduce the generalized factorization concept in $L_{2}(\pi)$.

We say that a matrix function $f \in\left[L_{\infty}(\pi)\right]_{n, n}$ admits a left (right) generalized factorization in $L_{2}(\pi)$ if it can be represented as

$$
f=f_{+} \Lambda f_{-} \quad\left(f=f_{-} \Lambda f_{+}\right)
$$

where

$$
f_{+}^{ \pm 1} \in\left[L_{2}^{+}(\pi)\right]_{n, n}, \quad f_{-}^{ \pm 1} \in\left[L_{2}^{-}(\pi)\right]_{n, n}, \quad \Lambda(t)=\operatorname{diag}\left\{t^{\varkappa_{i}}\right\}_{j=1}^{n},
$$

$\varkappa_{j} \in \mathbb{Z}, j=\overline{1, n}$, with $\varkappa_{1} \geq \varkappa_{2} \geq \ldots \geq \varkappa_{n}$, and $f_{+} P_{+} f_{-} I \quad\left(f_{-} P_{+} f_{+} I\right)$ represents a bounded linear operator in $\left[L_{2}(\pi)\right]_{n}$.

The number $\varkappa=\sum_{j=1}^{n} \varkappa_{j}$ is called the left (right) factorization index of the matrix function $f$. The integers $\varkappa_{j}$ are called the left (right) partial factorization indices of $f$. If $\varkappa_{j}=0, \forall j=\overline{1, n}$, then $f$ is said to admit a left (right) canonical generalized factorization.

\section{Remark 2.1.}

(i) A natural and nontrivial question is the relation between the left and right partial indices of a generalized factorization of a matrix function $f$. Whenever both left and right generalized factorizations in $L_{\infty}(\pi)$ exist, the left and right factorization index of the matrix function $f$ must be the same. That is, the factorization index of the scalar valued function det $f(t)$ coincides with the sum of all partial (both left and right) indices of $f$, the integer number $\varkappa$. It was proved in [29] that this relation is the only existing one between the sets of the left and right partial indices.

\footnotetext{
${ }^{4}$ The corresponding source code of [SInt] is available in the online version of [15].
} 
(ii) The left (right) partial indices $\varkappa_{i}$ are uniquely determined by the matrix function $f$, that is, in a factorization (5), the matrix $\Lambda$ is uniquely defined. However, this is not true for $f_{ \pm}$and the general relation between the factors of two distinct generalized factorizations of the same matrix function $f$ is described, for instance, in [31].

\subsubsection{Rational factorization.}

Any non-singular rational matrix function $f \in[\mathcal{R}(\pi)]_{n, n}$ admits a left (right) factorization of the form (5), where

For the particular rational scalar case

$$
f_{+}^{ \pm 1} \in\left[\mathcal{R}_{+}(\pi)\right]_{n, n}, \quad f_{-}^{ \pm 1} \in\left[\mathcal{R}_{-}(\pi)\right]_{n, n} .
$$

$$
\varkappa=z_{+}-p_{+},
$$

where $z_{+}$is the number of zeros of $f$ in $\pi_{+}$(with regard to their multiplicities) and $p_{+}$is the number of poles of $f$ in $\pi_{+}$(with regard to their multiplicities) (see, for instance, [16]).

From the computational point of view the rational factorization [ARFact-Scalar] and [ARFactMatrix] algorithms [16] can be used to compute explicit factorizations that allow to study the kernel of some classes of paired singular integral operators.

\section{Remark 2.2.}

(i) Note that the success of the [ARFact-Scalar] algorithm [16] depends on the possibility of finding solutions of polynomial equations. This can be a serious limitation when working with polynomials of the fifth degree or higher. However, even in this case, thanks to the symbolic and numeric capabilities of Mathematica, it is still possible to obtain an explicit, and for all purposes exact, rational factorization for the one-dimensional case. In fact, Mathematica uses Root objects to represent solutions of algebraic equations in one variable, when it is impossible to find explicit formulas for these solutions. The Root object is not a mere denoting symbol but rather an expression that can be symbolically manipulated and numerically evaluated with any desired precision. In particular, it is still possible to know if any given Root lies in $\pi$, in $\pi_{+}$, or in $\pi_{-}$, which is all the information the algorithm needs to compute the factors $f_{ \pm}$and the index $\varkappa$ (see Figure 12).

(ii) For the [ARFact-Matrix] algorithm [16], due to the complexity of the matrix case, the use of the Root objects to obtain an explicit factorization is not as feasible as before since the dimension of the matrix function is also a limiting factor, even when the entries of $f$ are rational functions with low degree polynomials [16]. However, the algorithm was designed in such a way as to have only an exact factorization as an output, that is, when it is not possible to determine a factorization due to the size of the matrix function or due to the degree of a polynomial, the algorithm does not give any factorization as an output. So, that there is no output problem related to the unstable case of a factorization (when the difference between the first and last partial index is greater than 1).

\subsubsection{Inner-outer factorization.}

Another factorization concept that is very important to study the spectrum and the kernel of several classes of singular integral operators is the inner-outer factorization of bounded analytic functions defined in the interior of the unit circle.

The generalized factorization algorithm [AFact] uses the inner-outer factorization concept to factorize some special classes of essentially bounded matrix functions [11, 17]. The classes of matrix functions that can be factorized by this analytic algorithm are closely related to the solution of the non-linear Schrödinger equation (see, for instance, [27]), to the generalized Riemann-Hilbert problem (see, for instance, [20, 21, 41, 42]), and to the study of singular integral operators related with Hankel operators [18].

Let $H_{r, \theta}$ be the subset of $H_{\infty}(\pi)$ containing the functions that can be represented as the product of a rational outer function $r$ (i.e, $r$ has all the zeros and poles in $\pi_{-}$) and an inner function ${ }^{5} \theta$ (i.e., $\theta$ is a bounded analytic function on $\pi_{+}$such that its modulus is equal to one a.e. on $\pi$ ).

\footnotetext{
${ }^{5}$ Some of our analytic algorithms [15, 17, 21, 23] provide us extra information about the class of inner functions.
} 
In Section 5 it is explained how the dimension of the kernel of some classes of paired singular integral operators is related with the dimension of the kernel of some classes of singular integral operators connected with Hankel operators with coefficients in $H_{r, \theta}$.

\section{On the kernel of paired singular integral operators}

In this section we will see how the study of the factorability, of scalar and matrix functions, is related to the study of the kernel of the paired singular integral operators $T_{\{a, b\}}$ and $\widetilde{T}_{\{a, b\}}$ defined in (3) and (4), for the one-dimensional and matrix case.

3.1. Some relations between the dimensions of the kernels of $T_{\{a, b\}}$ and $\widetilde{T}_{\{a, b\}}$

Let $e$ denote the $n \times n$ identity matrix function.

Concerning the questions of invertibility or Fredholm theory the existing references, up to our knowledge, only consider matrix paired singular integral operators in the form $T_{\{a, e\}}=a P_{+}+P_{-}$or $\widetilde{T}_{\{e, a\}}=P_{+}+P_{-} a I$ (see, for instance, $[10,43]$ ) or the one-dimensional case (see, for instance, [33, 34]).

With the same ideas used in the proof of Theorem 2.2 in [24], there arises the following result ${ }^{6}$

[12] about the kernel of the operators $T_{\{a, b\}}$ and $\widetilde{T}_{\{a, b\}}$ that is a generalization of the known result for the one-dimensional case (see, for instance, [33]).

Theorem 3.1. Let $a^{ \pm 1}, b \in\left[L_{\infty}(\pi)\right]_{n, n}$. If $a b=b a$, then

$$
\operatorname{dim} \operatorname{ker} T_{\{a, b\}}=\operatorname{dim} \operatorname{ker} \widetilde{T}_{\{a, b\}} .
$$

Remark 3.1. The invertible operator $W_{2}$ considered in the proof of Theorem 3.1 can be used to compute the $\widetilde{T}_{\{a, b\}}$ kernel's through the kernel of $T_{\{a, b\}}$. In fact, a base of the kernel of $\widetilde{T}_{\{a, b\}}$ can be constructed by applying the operator $W_{2}^{-1}$ to the elements of a base of the kernel of $T_{\{a, b\}}$. In a similar way, the $T_{\{a, b\}}$ kernel's can be computed through the kernel of $\widetilde{T}_{\{a, b\}}$ applying the operator $W_{2}$ to its base.

In the case when $a b \neq b a$, the equality (8) is not necessarily satisfied. In fact, several interesting examples where $\operatorname{dim} \operatorname{ker}\left(T_{\{a, b\}}\right) \neq \operatorname{dim} \operatorname{ker}\left(\widetilde{T}_{\{a, b\}}\right)$ can be easily constructed with the use of the computer algebra system Mathematica (see Examples 4.5 and 4.6). Obviously, in the scalar case the equality (8) is always satisfied.

\subsection{The rule of the factorization partial indices in the study of the kernel of $T_{\{a, b\}}$ and $\widetilde{T}_{\{a, b\}}$}

Now we will see how the computation of the partial indices of a matrix function is related with the study of the kernel of paired singular integral operators $T_{\{a, b\}}$ and $\widetilde{T}_{\{a, b\}}$ defined by (3) and (4). In particular, since the calculation of the factorization index is always possible (see Remark 2.2) in the scalar rational case by using the [ARFact-Scalar] algorithm, the dimension of the kernel of the onedimensional paired singular integral operators $T_{\{a, b\}}$ and $\widetilde{T}_{\{a, b\}}$ can be computed using the computer algebra system Mathematica (see subsection 4.1 [ADimKerPaired-Scalar] algorithm).

\subsubsection{The matrix case.}

The next result ${ }^{7}$ on the dimension of the kernel of the paired singular integral operator $T_{\{a, b\}}$ defined by (3) is well known (see, for instance, [42]).

Theorem 3.2. Let $a, b \in\left[L_{\infty}(\pi)\right]_{n, n}$. If $a^{-1} \in\left[L_{\infty}(\pi)\right]_{n, n}$ and the matrix function $a^{-1} b$ admits a left generalized factorization (5) in $L_{2}(\pi)$, then $\operatorname{dim} \operatorname{ker} T_{\{a, b\}}<\infty$. In this case, the dimension of the kernel of the paired singular integral operator $T_{\{a, b\}}$ corresponds to the sum of the left positive partial indices of $a^{-1} b$.

${ }^{6} \mathrm{An}$ analogous result can also be stated for the case when $b^{-1} \in\left[L_{\infty}(\mathbb{\pi})\right]_{n, n}$ changing $\left(I \pm P_{\mp} a^{-1} b P_{ \pm}\right) a^{\mp 1} I$ by $\left(I \pm P_{ \pm} a b^{-1} P_{\mp}\right) b^{\mp 1} I$.

${ }^{7} \mathrm{~A}$ similar result can be obtained for the case when $b^{-1} \in\left[L_{\infty}(\mathbb{\pi})\right]_{n, n}$ and the matrix function $b^{-1} a$ admits a right generalized factorization in $L_{2}(\pi)$. In this case, the dimension of the kernel of the paired singular integral operator $T_{\{a, b\}}$ corresponds to the sum of the modulus of the right negative partial indices of $b^{-1} a$. 
Based on the same ideas, similar results ${ }^{8}$ can be formulated for the singular integral operator $\widetilde{T}_{\{a, b\}}$ defined by (4).

Theorem 3.3. Let $a, b \in\left[L_{\infty}(\pi)\right]_{n, n}$. If $a^{-1} \in\left[L_{\infty}(\mathbb{\pi})\right]_{n, n}$ and the matrix function $b^{-1}$ admits a left generalized factorization (5) in $L_{2}(\pi)$, then $\operatorname{dim} \operatorname{ker} \widetilde{T}_{\{a, b\}}<\infty$. In this case, the dimension of the kernel of the paired singular integral operator $\widetilde{T}_{\{a, b\}}$ corresponds to the sum of the left positive partial indices of $b a^{-1}$.

The next result ${ }^{9}$ is a complement to the Theorem 3.2 .

Theorem 3.4. Let $a, b \in\left[L_{\infty}(\pi)\right]_{n, n}$. If $a^{-1} \in\left[L_{\infty}(\mathbb{\pi})\right]_{n, n}$ and the matrix function $a^{-1} b$ admits a left generalized factorization $(5)$ in $L_{2}(\pi)$,

$$
a^{-1} b=f=f_{+} \Lambda f_{-}
$$

then

$$
\operatorname{ker} T_{\{a, b\}}=\left\{\varphi: \varphi=\left[f_{+}-f_{-}^{-1} \Lambda^{-1}\right] P\right\},
$$

where $P=\left(P_{\varkappa_{1}-1}, \cdots, P_{\varkappa_{n}-1}\right), P_{\varkappa_{j}-1}(t)$ denotes an arbitrary polynomial of degree at most $\varkappa_{j}-1$.

Analogously, formulas for the kernel of the operator $\widetilde{T}_{\{a, b\}}$, defined by (4), can be obtained using a left generalized factorization of the matrix function $b a^{-1}$ or a right generalized factorization of the matrix function $a b^{-1}$.

So, in the rational case, the [ARFact-Matrix] algorithm can be used to compute the kernel of $T_{\{a, b\}}$ by computing the factors of a left factorization (5) of the matrix function $a^{-1} b$ (or the factors of a right factorization of $b^{-1} a$ ). For the operator $\widetilde{T}_{\{a, b\}}$ the [ARFact-Matrix] algorithm compute a left factorization (5) of the matrix function $b a^{-1}$ (or the factors of a right factorization of $a b^{-1}$ ) (see subsection 4.3 [ADimKerPaired-Matrix] algorithm).

The hermitian case. Since the study of paired singular integral operators with hermitian matrix coefficients is an important line of research in operator theory (see Section 5) it's interesting to see what is the effect of the Theorems 3.2 and 3.3 in this special class of paired singular integral operators. We get the following results on the kernels of the operators $T_{\{a, b\}}$ and $\widetilde{T}_{\{a, b\}}$.

Theorem 3.5. Let $a^{ \pm 1}, b \in\left[L_{\infty}(\pi)\right]_{2 n, 2 n}$. If $a^{-1} b$ is an hermitian matrix function that admits a left generalized factorization (5) in $L_{2}(\pi)$, then

$$
\operatorname{dim} \operatorname{ker} T_{\{a, b\}}=\sum_{i=1}^{n} \varkappa_{i},
$$

where $\varkappa_{i}, i=\overline{1, n}$ are the left partial indices of $a^{-1} b$.

Proof. If $a^{-1} b$ is a left factorable hermitian matrix function its left partial indices are connected via the relations [42]

$$
\varkappa_{j}+\varkappa_{2 n-j+1}=0, \forall j=\overline{1,2 n} .
$$

Since $\varkappa_{1} \geq \varkappa_{2} \geq \ldots \geq \varkappa_{2 n}$, we get $(9)$.

Theorem 3.6. Let $a^{ \pm 1}, b \in\left[L_{\infty}(\pi)\right]_{2 n, 2 n}$. If $b a^{-1}$ is an hermitian matrix function that admits a left generalized factorization (5) in $L_{2}(\pi)$, then

$$
\operatorname{dim} \operatorname{ker} \widetilde{T}_{\{a, b\}}=\sum_{i=1}^{n} \widetilde{\varkappa}_{i},
$$

\footnotetext{
${ }^{8}$ Similar results can be obtained for the case when $b^{-1} \in\left[L_{\infty}(\pi)\right]_{n, n}$ and the matrix function $a b^{-1}$ admits a right generalized factorization in $L_{2}(\pi)$. In this case, the dimension of the kernel of the paired singular integral operator corresponds to the sum of the modulus of the right negative partial indices of $a b^{-1}$.

${ }^{9} \mathrm{~A}$ similar result can be obtained for the case when $b^{-1} \in\left[L_{\infty}(\mathbb{T})\right]_{n, n}$ and the matrix function $b^{-1} a$ admits a right generalized factorization in $L_{2}(\pi)$.
} 
where $\tilde{\varkappa}_{i}, i=\overline{1, n}$ are the left partial indices of $b a^{-1}$.

\subsubsection{The one-dimensional case.}

Let us now consider the one-dimensional paired singular integral operators $T_{\{a, b\}}$ and $\widetilde{T}_{\{a, b\}}$.

From Theorem 3.2, Theorem 3.4, and the similar results for the operator $\widetilde{T}_{\{a, b\}}$, we get the following result ${ }^{10}$ that allows us to compute the kernels of the operators $T_{\{a, b\}}$ and $\widetilde{T}_{\{a, b\}}$ defined by (3) and (4).

Theorem 3.7. Let $a^{ \pm 1}, b \in L_{\infty}(\mathbb{\pi})$ and assume that the function $f=a^{-1} b$ admits a generalized factorization $f(t)=f_{+}(t) t^{\varkappa} f_{-}(t)$ in $L_{2}(\pi)$. In this case,

$$
\operatorname{dim} \operatorname{ker} T_{\{a, b\}}=\operatorname{dim} \operatorname{ker} \widetilde{T}_{\{a, b\}}=\max \{0, \varkappa\} .
$$

Furthermore, for $\varkappa>0$ and $g(t)=f_{+}(t)-f_{-}^{-1}(t) t^{-\varkappa}$,

$$
\operatorname{ker} T_{\{a, b\}}=\operatorname{span}\left\{g, g t, \cdots, g t^{\varkappa-1}\right\}
$$

and

$$
\operatorname{ker} \widetilde{T}_{\{a, b\}}=\operatorname{span}\left\{a^{-1} f_{-}^{-1} t^{-\varkappa}, a^{-1} f_{-}^{-1} t^{-\varkappa+1}, \cdots, a^{-1} f_{-}^{-1} t^{-1}\right\} .
$$

So, in the rational case it is always possible to compute the dimension of the kernel of $T_{\{a, b\}}$ and $\widetilde{T}_{\{a, b\}}$ since the factorization index of a factorable function $a^{-1} b$ can be computed (when it exists) by using the [ARFact-Scalar] algorithm [16]. Furthermore, $\operatorname{ker} T_{\{a, b\}}$ and $\operatorname{ker} \widetilde{T}_{\{a, b\}}$ can be effectively computed since the [ARFact-Scalar] algorithm also computes the factors $f_{ \pm}$of a factorization (5). On the other hand, the [SInt] algorithm [15] can be used to compute the kernel of $\widetilde{T}_{\{a, b\}}$ through the kernel of $T_{\{a, b\}}$, or vice versa (see Remark 3.1).

\subsubsection{How to relate one-dimensional and matrix cases.}

This subsection is dedicated to some results ${ }^{11}$ that relate the dimension of the kernel of a onedimensional paired singular integral operator to the dimension of the kernel of a paired singular integral operator with matrix coefficients.

The next result [12], which comes directly from Remark 2.1, relates a generalized factorization in $L_{2}(\pi)$ of a factorable matrix function $A$ to a generalized factorization of its determinant.

Theorem 3.8. Let $f \in\left[L_{\infty}(\pi)\right]_{n, n}$ be a non-singular (left and right) factorable matrix function. If $\operatorname{det} f(t)$ admits a non-canonical generalized factorization in $L_{2}(\pi)$, then $f$ admits a non-canonical (left and right) generalized factorization in $L_{2}(\pi)$.

The importance of Theorem 3.8 increases with the development of the use of symbolic computation within operator theory. In fact, using algorithms to compute the factorization index of scalar rational functions [16], it is possible to conclude, for certain classes of matrix functions, its kind of factorization. For instance, in the rational case, since it is always possible to determine the factorization index $\varkappa$ (when it exists) of a scalar rational function, if $\varkappa \neq 0$, we can conclude that the corresponded matrix function (when factorable) admits a non-canonical factorization.

Example 3.1. Let us consider the non-singular matrix function belonging to $[\mathcal{R}(\pi)]_{3,3}$,

$$
f(t)=\left(\begin{array}{ccc}
t-2 & 1 & 1 \\
2 & t & t+1 \\
2 t-1 & 1 & -1
\end{array}\right) .
$$

In this case, $\operatorname{det} f(t)=-2 t^{2}+5 t+5$. Using the [ARFact-Scalar] algorithm we get the factorization index of detf, $\varkappa=1$. Since $\varkappa \neq 0$, by Theorem 3.8 the matrix function $f$ admits a non-canonical left factorization.

10 [33] contains formulas to compute the kernels of $T_{\{a, b\}}$ and $\widetilde{T}_{\{a, b\}}$ using the factors of a factorization of the function $a b^{-1}$.

${ }^{11}$ Similar results can be obtained for the case when the matrix function $b a^{-1}$ admits a left generalized factorization in $L_{2}(\pi)$. 
Example 3.1.1 Right factorization

The [ARFact-Matrix] algorithm computes the right partial indices of $f: \varkappa_{1}=1, \varkappa_{2}=0, \varkappa_{3}=0$ (see Figure 2).

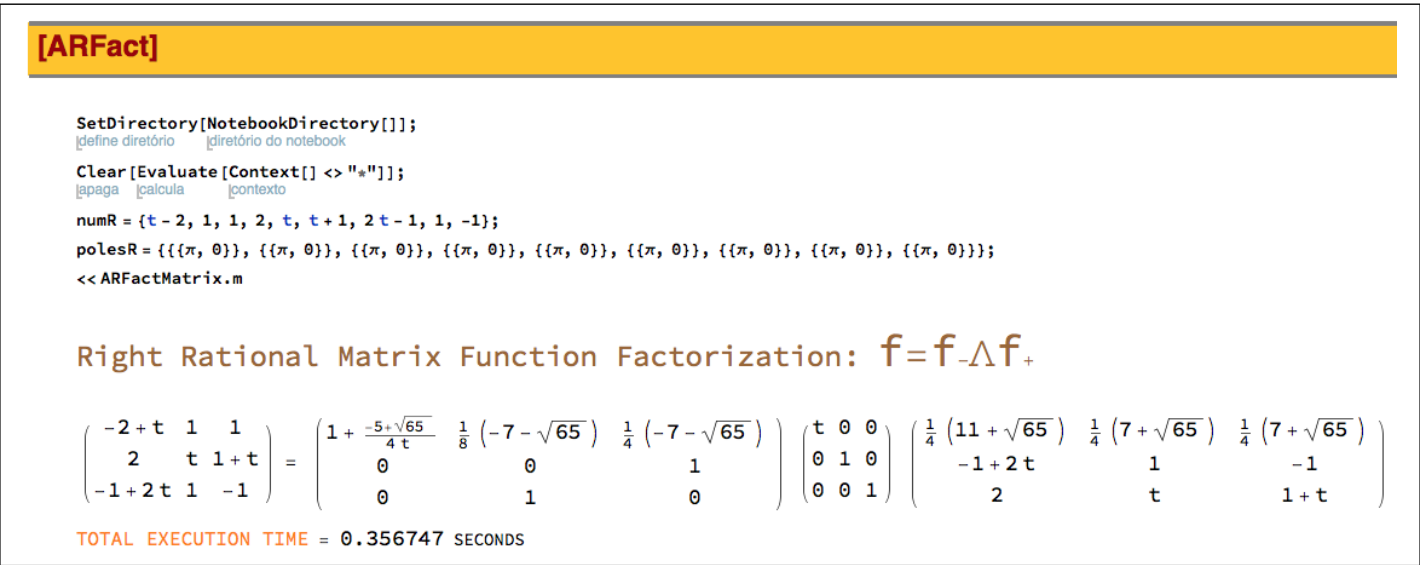

FIgURE 2. Right factorization of $f$ computed with the [ARFact-Matrix] algorithm.

Example 3.1.2 Left factorization

The [ARFact-Matrix] algorithm computes the left partial indices of $f: \varkappa_{1}=1, \varkappa_{2}=0, \varkappa_{3}=0$ (see Figure 3).

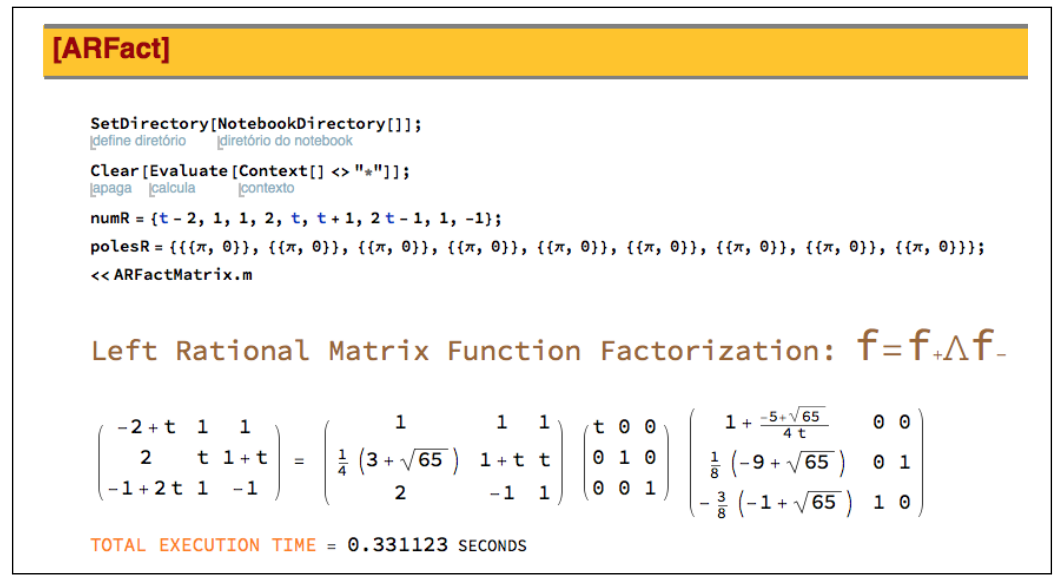

FIGURE 3. Left factorization of $f$ computed with the [ARFact-Matrix] algorithm.

Remark 3.2. In Example 3.1 the left and right partial indices are equal.

Example 3.2. Let us consider the non-singular matrix function belonging to $[\mathcal{R}(\pi)]_{3,3}$,

$$
f(t)=\left(\begin{array}{ccc}
1+\frac{1}{t-3} & t(2 t-7 i)-3 & 0 \\
(t-3)(2 t-1)(3 t+1) & 0 & 0 \\
\frac{(2 t-1)^{2}}{t-3 i} & 0 & \frac{-1}{(t-3)^{2}}
\end{array}\right) .
$$

In this case, $\operatorname{det} f(t)=\frac{(2 t-1)(3 t+1)\left(2 t^{4}-(6+13 i) t^{3}-(24-39 i) t^{2}+(72+9 i) t-27 i\right)}{(t-3)^{2}(t-3 i)}$. Using the [ARFact-Scalar] algorithm we get the factorization index of detf, $\varkappa=3$. Since $\varkappa \neq 0$, by Theorem 3.8 the matrix function $f$ admits a non-canonical left factorization. 
Example 3.2.1 Right factorization

The [ARFact-Matrix] algorithm computes the right partial indices of $f: \varkappa_{1}=2, \varkappa_{2}=1, \varkappa_{3}=0$ (see Figure 4).

\section{[ARFact]}

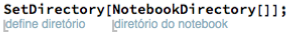

Clear [Evaluate [Context [] $<>" * "]]$;

numR $=\left\{t-2,(2 t-i)(t-3 i), \theta,(t-3)(2 t-1)(3 t+1), \theta, \theta,(2 t-1)^{2}, \theta,-1\right\} ;$

polesR $=\{\{\{3,1\}\},\{\{\pi, 0\}\},\{\{\pi, 0\}\},\{\{\pi, 0\}\},\{\{\pi, 0\}\},\{\{\pi, 0\}\},\{\{3$ i, 1$\}\},\{\{\pi, 0\}\},\{\{3,2\}\}\} ;$

$\ll$ ARFactMatrix.m

Right Rational Matrix Function Factorization: $f=f-\Lambda f$

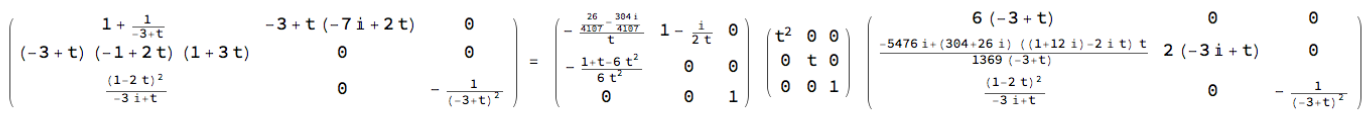

TOTAL EXECUTION TIME $=0.351864$ SECONDS

FigURE 4. Right factorization of $f$ computed with the [ARFact-Matrix] algorithm.

\section{Example 3.2.2 Left factorization}

The [ARFact-Matrix] algorithm computes the left partial indices of $f: \varkappa_{1}=1, \varkappa_{2}=1, \varkappa_{3}=1$ (see Figure 5).

\section{[ARFact]}

SetDirectory [NotebookDirectory []];

Clear [Evaluate [Context [] <>"*"]];

numR $=\left\{t-2,(2 t-i)(t-3 i), \theta,(t-3)(2 t-1)(3 t+1), \theta, \theta,(2 t-1)^{2}, \theta,-1\right\} ;$

polesR $=\{\{\{3,1\}\},\{\{\pi, 0\}\},\{\{\pi, 0\}\},\{\{\pi, 0\}\},\{\{\pi, 0\}\},\{\{\pi, 0\}\},\{\{3 i, 1\}\},\{\{\pi, 0\}\},\{\{3,2\}\}\} ;$

$\ll$ ARFactMatrix.m

Left Rational Matrix Function Factorization: $f=f+\Lambda f$.

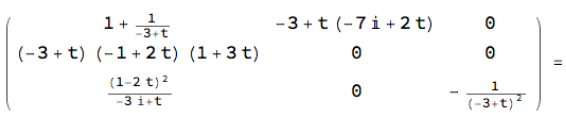

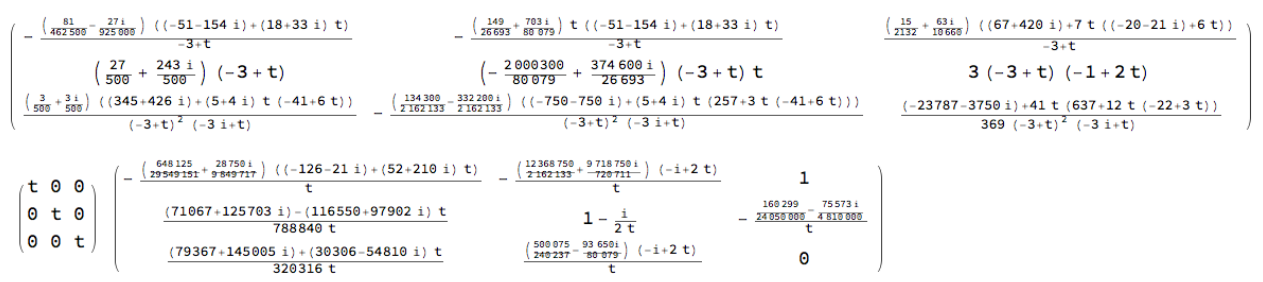

TOTAL EXECUTION TIME $=1.434402$ SECONDS

FIgURE 5. Left factorization of $f$ computed with the [ARFact-Matrix] algorithm.

Remark 3.3. In Example 3.2 the left and right partial indices are not equal. 
Example 3.3. Let us consider the non-singular matrix function belonging to $[\mathcal{R}(\pi)]_{7,7}$,

$$
f(t)=\left(\begin{array}{ccccccc}
\frac{1}{(t-2) t} & \frac{i(4 t-1)}{t-7 i} & 0 & \frac{1}{t-2} & 0 & 0 & \frac{1}{t-2} \\
\frac{t+1}{4 t-1} & 2 i & \frac{i(t+1)}{4 t-1} & 0 & 0 & t^{-1} & 0 \\
\frac{i}{(t-2) t} & \frac{1-4 t}{t-7 i} & 0 & \frac{i(t-1)}{t-2} & 0 & 0 & \frac{1}{t-2} \\
-1 & 0 & -i & 0 & 0 & 1 & 0 \\
\frac{t-2}{t} & 0 & 0 & 1 & 0 & t^{-2} & 0 \\
t-2 & \frac{4 t-1}{t} & 0 & 0 & 0 & 0 & 0 \\
-\frac{1}{(t-2) t^{2}} & -\frac{i(4 t-1)}{(t-7 i) t} & 0 & -\frac{1}{(t-2) t} & t & 0 & -\frac{1}{(t-2) t}
\end{array}\right) .
$$

In this case, $\operatorname{det} f(t)=\left(t^{2}+(5+2 i) t-1\right) t^{-2}$. Using the [ARFact-Scalar] algorithm we get the factorization index of detf, $\varkappa=-1$. Since $\varkappa \neq 0$, by Theorem 3.8 the matrix function $f$ admits a non-canonical left factorization.

In this example the [ARFact-Matrix] algorithm failed (see Figure 6) to determine the left partial indices due to the high degree of the polynomials of the entries of the matrix function. However, using the Theorem 3.8 , and the [ARFact-Scalar] algorithm, it is possible to know that the matrix $f$ admits a non-canonical left factorization.

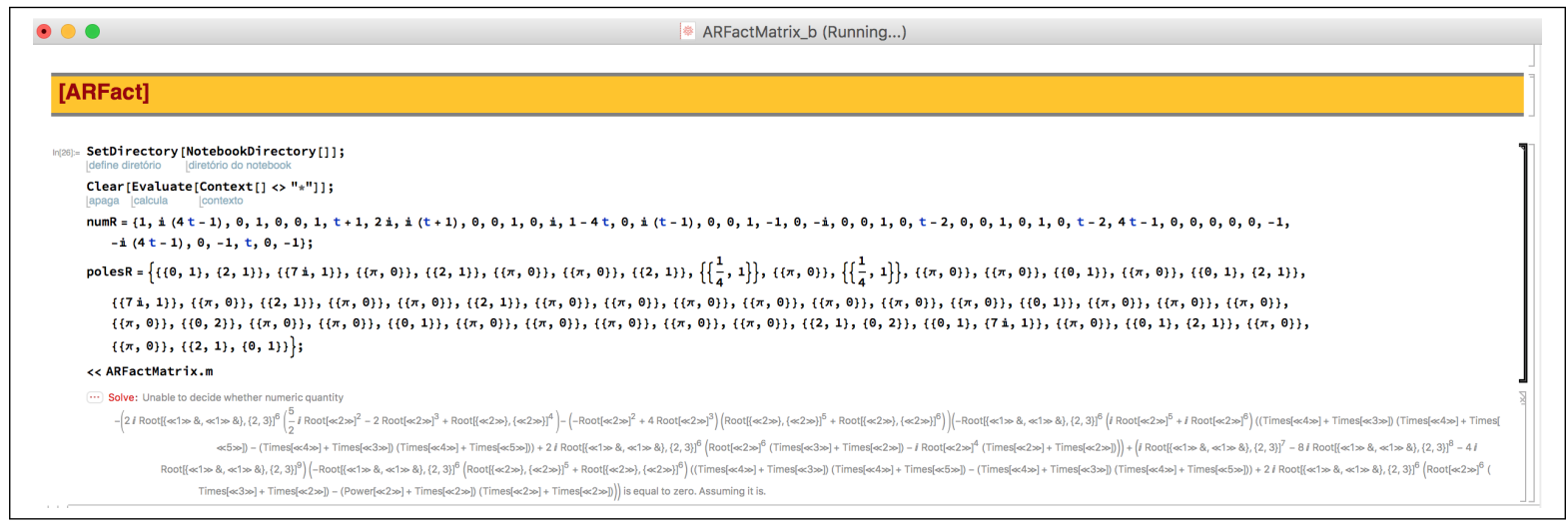

Figure 6. The [ARFact-Matrix] algorithm failed to compute a left factorization of $f$.

Remark 3.4. If the scalar function det $f(t)$ admits a canonical generalized factorization in $L_{2}(\pi)$, then a factorable (left and right) matrix function $f$ can admit a (left and right) canonical or a (left and right) non-canonical generalized factorization in $L_{2}(\pi)$.

Remark 3.4 can be easily illustrated using the algorithms [ARFact-Matrix] and [AFact].

Example 3.4. Let us consider the factorable matrix function (see, for instance, [17]) belonging to $\left[L_{\infty}(\pi)\right]_{2,2}$,

$$
f(t)=\left(\begin{array}{cc}
1 & (t-2) \theta(t) \\
(1-2 t) t^{-1} \bar{\theta}(t) & \left(-2 t^{2}+4 t-2\right) t^{-1}
\end{array}\right)
$$

where $\theta$ is an arbitrary inner function.

Since $\operatorname{det} f(t)=-1$ it is obvious that $\varkappa=0, \forall \theta$.

Example 3.4.1. Right factorization

$A$ right canonical factorization of $f$ can easily be obtained using some formulas presented in [19]

$$
\left(\begin{array}{cc}
1 & (t-2) \theta(t) \\
(1-2 t) t^{-1} \bar{\theta}(t) & \left(-2 t^{2}+4 t-2\right) t^{-1}
\end{array}\right)=\left(\begin{array}{cc}
1 & 0 \\
(1-2 t) t^{-1} \bar{\theta}(t) & -1
\end{array}\right)\left(\begin{array}{cc}
1 & (t-2) \theta(t) \\
0 & 1
\end{array}\right) .
$$


Example 3.4.2. Left factorization

In this case, $f$ is an hermitian matrix function, for all inner function $\theta$. So, using relation (10) we get that $\varkappa_{1}+\varkappa_{2}=0$. That is, $f$ admits a left canonical generalized factorization or a non-canonical generalized factorization with symmetric left partial indices.

Example 3.4.2.a) Case $\theta^{\prime}(1) \neq 0$

Using the [AFact] algorithm we get the left partial indices $\varkappa_{1}=0$ and $\varkappa_{2}=0$, for all inner function $\theta$ such that $\theta^{\prime}(1) \neq 0$ (see Figure 7 ).

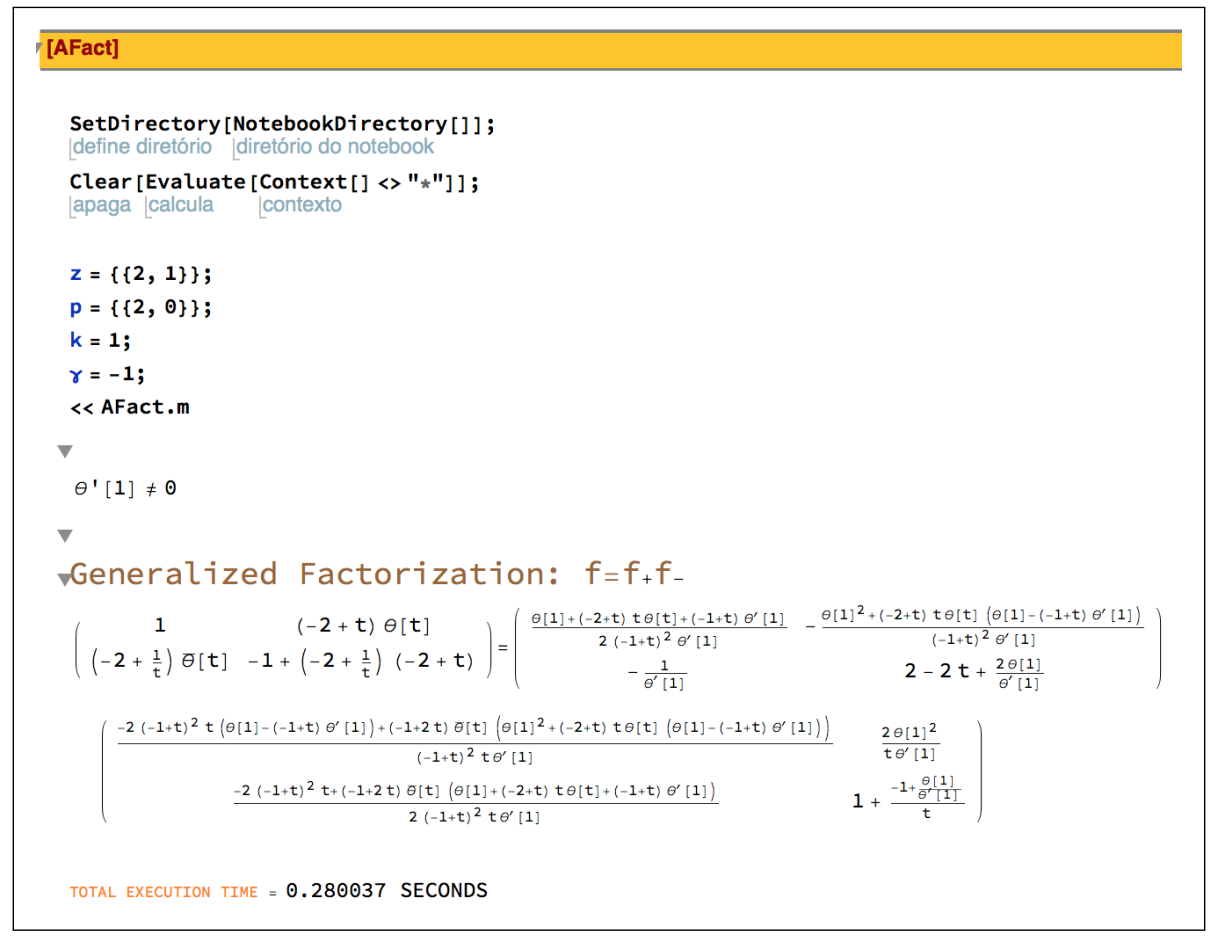

Figure 7. Left generalized factorization of $f$ computed with the [AFact] algorithm.

Example 3.4.2.b) Case $\theta(t)=1$

Using the [ARFact-Matrix], or the [AFact], algorithm we get the left partial indices $\varkappa_{1}=1$ and $\varkappa_{2}=-1$ (see Figure 8 and Figure 9).

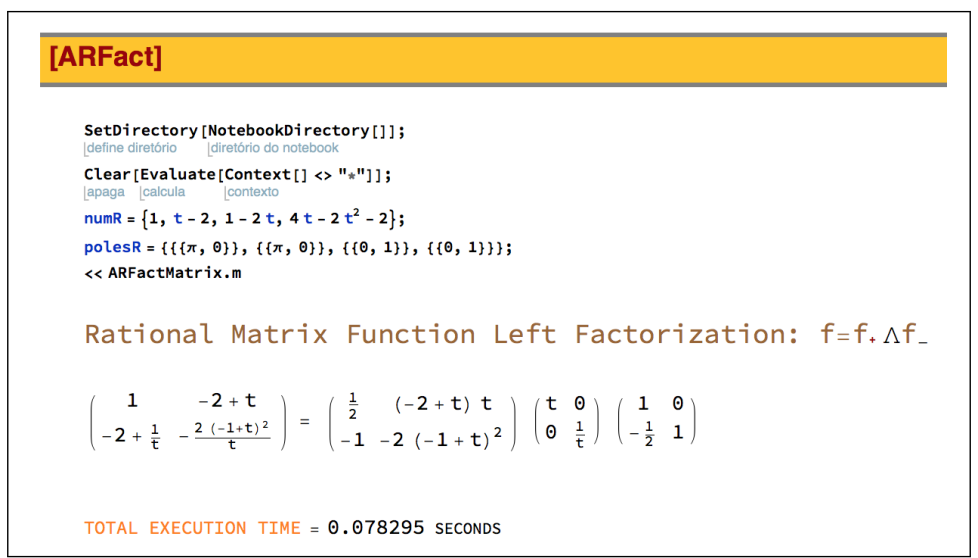

FIGURE 8. Left generalized factorization of $f$ computed with the [ARFact-Matrix] algorithm. 


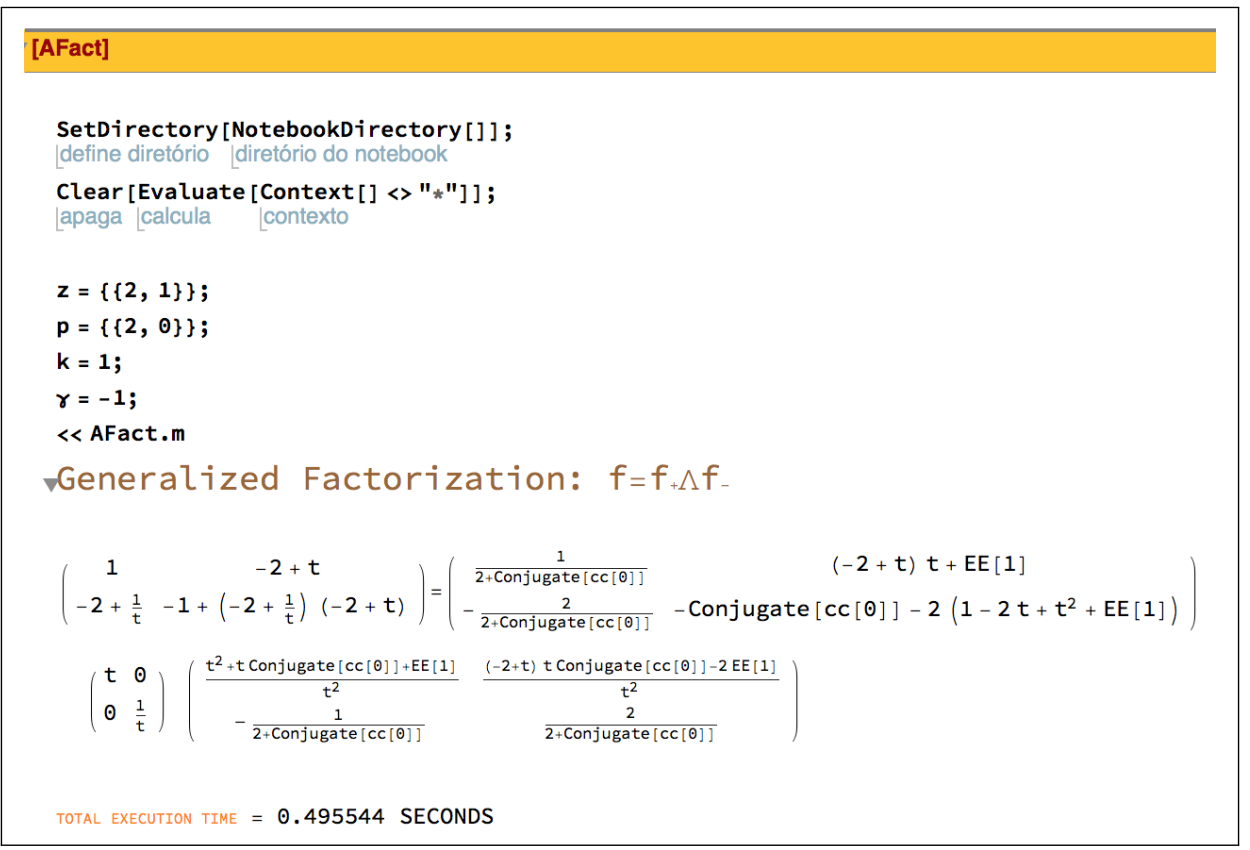

Figure 9. Left generalized factorization of $f$ computed with the [AFact] algorithm.

The next result [12] relates the dimension of the kernel of a one-dimensional paired singular integral operator with the dimension of the kernel of a paired singular integral operator with matrix coefficients.

Theorem 3.9. Let $a^{ \pm 1}, b \in\left[L_{\infty}(\pi)\right]_{n, n}$ such that $f=a^{-1} b$ admits a left generalized factorization in $L_{2}(\pi)$. If $\operatorname{dim} \operatorname{ker}\left(P_{+}+(\operatorname{det} f) P_{-}\right)>0$, then $\left.\operatorname{dim} \operatorname{ker} T_{\{a, b\}}\right)>0$.

Similar results were obtained for the operator $\widetilde{T}_{\{a, b\}}$ considering left generalized factorization in $L_{2}(\pi)$ for the matrix function $b a^{-1}$.

Remark 3.5. Let $a^{ \pm 1}, b^{ \pm 1} \in\left[L_{\infty}(\pi)\right]_{n, n}$ such that $f=a^{-1} b$ and $g=b a^{-1}$ admits left generalized factorizations in $L_{2}(\pi)$. If the operators $P_{+}+(\operatorname{det} f) P_{-}$and $P_{+}+(\operatorname{det} g) P_{-}$have trivial kernels, then $T_{\{a, b\}}$ and $\widetilde{T}_{\{a, b\}}$ can have trivial or nontrivial kernels (see Examples 4.5 and 4.6 ).

\section{Exploring the kernel of paired singular integral operators with symbolic computation techniques}

In this section it is described how the symbolic and numeric computation capabilities of a computer algebra system allow us to study the kernel of several classes of singular integral operators. The analytical algorithms [ADimKerPaired-Scalar], AKerPaired-Scalar], and [ADimKerPaired-Matrix] are presented. The section also contains nontrivial rational examples computed with the use of a computer algebra system.

\section{1. [ADimKerPaired-Scalar] algorithm}

In this subsection it is shown how the symbolic and numeric computation capabilities of Mathematica can be used to design and implement an algorithm to compute the dimension of the kernel of some classes of one-dimensional paired singular integral operators.

The [ADimKerPaired-Scalar] computes the dimension of the kernel of one-dimensional paired singular integral operators of the form

$$
T_{\{a, b\}}=a P_{+}+b P_{-} \quad \text { and } \quad \widetilde{T}_{\{a, b\}}=P_{+} a I+P_{-} b I,
$$


defined in (3) and (4), respectively, with non-singular $a, b \in \mathcal{R}(\pi)$. The implementation of this algorithm with the Mathematica software system makes the results of lengthy and complex calculations available in a simple way.

This analytical algorithm has a rather simple structure (see Figure 10) since the knowledge of the factorization index $\varkappa$ of a non-singular scalar rational function, that can be determined by formula (7), is the only information the algorithm needs to determine the dimension of the kernel of the operators $T_{\{a, b\}}$ and $\widetilde{T}_{\{a, b\}}$ (see Theorem 3.7). In addition, the symbolic computation capabilities of Mathematica, and the pretty-print functionality ${ }^{12}$, allow the [ADimKerPaired-Scalar] code to be very simple and syntactically similar to its analytical counterpart.

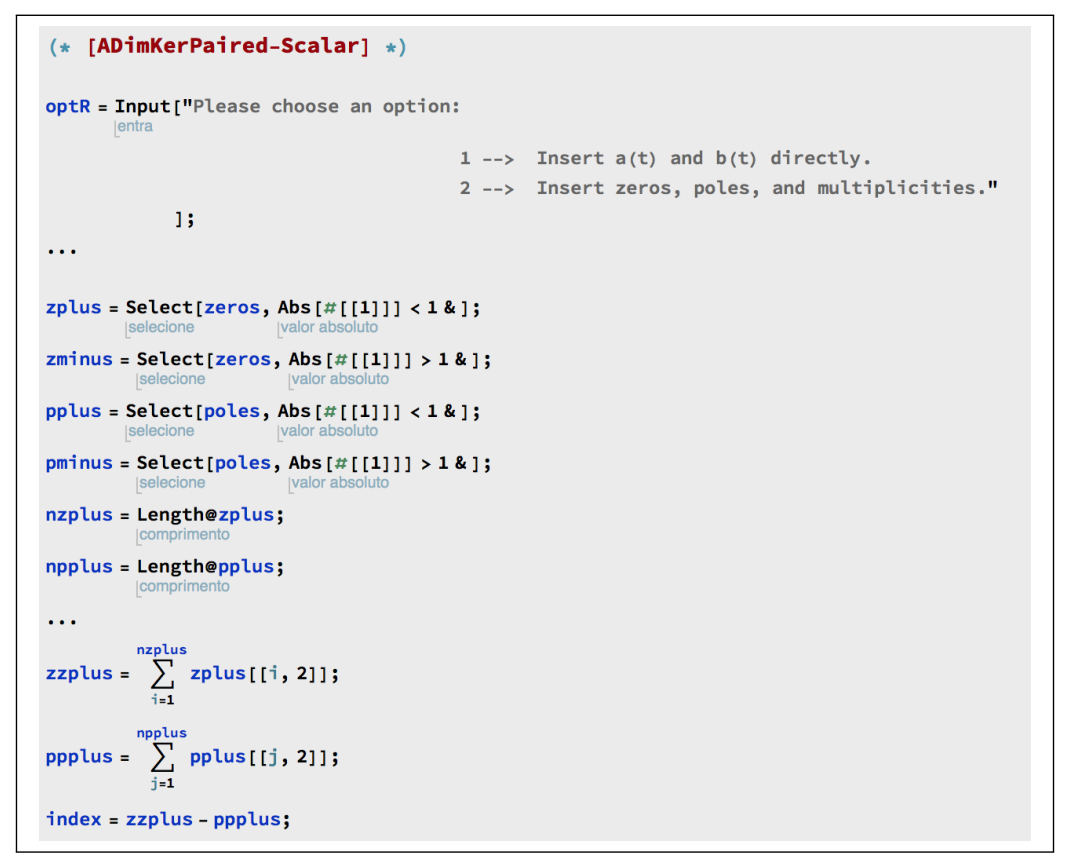

FiguRE 10. Snippet of the Mathematica's code responsible for the computation of the factorization index $\varkappa$ in the [ADimKerPaired-Scalar] algorithm.

The [ADimKerPaired-Scalar] algorithm can be applied to any one-dimensional paired singular integral operator of classes (3) and (4), with non-singular rational coefficients defined in $\pi$. There are two options to input $a(t)$ and $b(t)$ :

1. Insert $a(t)$ and $b(t)$ directly.

2. Insert zeros, poles, and multiplicities.

For each pair of inputed functions $a, b$ the [ADimKerPaired-Scalar] algorithm gives one of the following outputs:

$$
\begin{aligned}
& \text { [Output 1] } \operatorname{dim} \operatorname{ker} T_{\{a, b\}}=0 \text { and } \operatorname{dim} \operatorname{ker} \widetilde{T}_{\{a, b\}}=0 \\
& \text { [Output 2] } \operatorname{dim} \operatorname{ker} T_{\{a, b\}}=\varkappa \text { and } \operatorname{dim} \operatorname{ker} \widetilde{T}_{\{a, b\}}=\varkappa
\end{aligned}
$$

where $\varkappa$ is the factorization index $\varkappa$ of the function ${ }^{13} a^{-1} b$.

Figure 11 presents the pseudo code of the [ADimKerPaired-Scalar] algorithm.

\footnotetext{
${ }^{12}$ The pretty-print functionality allows to write on the computer screen scientific formulas in the traditional format, as if one was using pencil and paper.

${ }^{13} \mathrm{~A}$ similar algorithm can be described considering a factorization of the function $a b^{-1}$.
} 


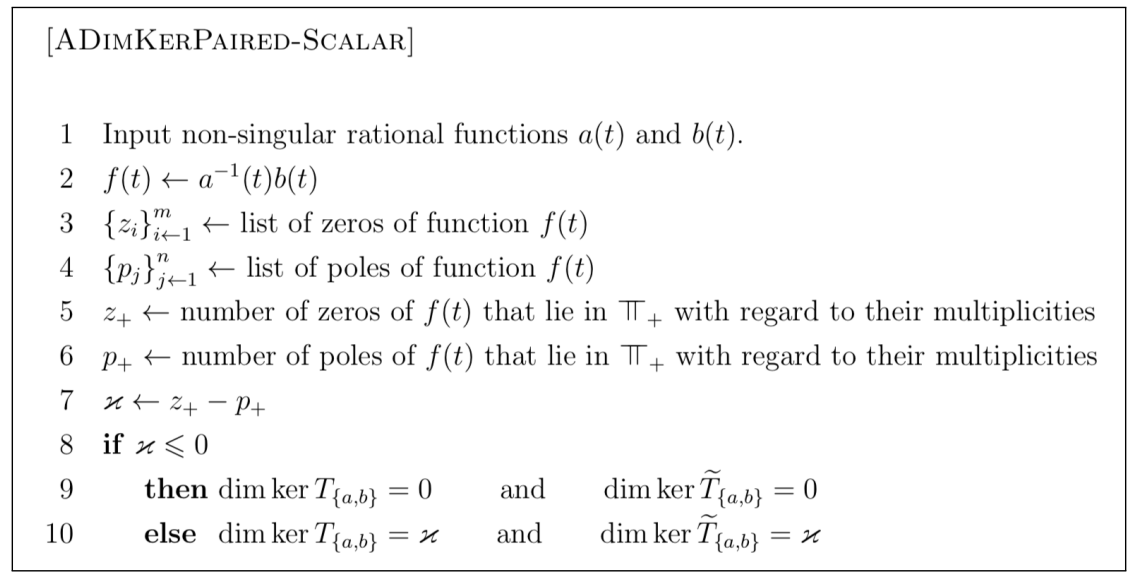

Figure 11. Pseudo code of the [ADimKerPaired-Scalar] algorithm.

The analysis of the pseudo code reveals that one main step in this algorithm is the computation of the zeros and poles (with regard to their multiplicities), of the non-singular rational function

$$
f(t)=a^{-1}(t) b(t)
$$

and whether they lie in $\pi_{ \pm}$(also verify if they do not lie in $\pi$ since it is a necessary condition for $f$ to be a factorable function). So, the success of the [ADimKerPaired-Scalar] algorithm depends on the possibility of finding those zeros and poles by solving polynomial equations. This can be a serious limitation when working with polynomials of the fifth degree or higher. However, even in this case, thanks to the symbolic and numeric capabilities of Mathematica, it is still possible to compute the dimension of the kernel of the operators (3) and (4). In fact, Mathematica uses Root objects to represent solutions of algebraic equations in one variable, when it is impossible to find explicit formulas for these solutions. The Root object is not a mere denoting symbol but rather an expression that can be symbolically manipulated and numerically evaluated with any desired precision. In particular, it is still possible to know if any given Root lies in $\pi, \pi_{+}$, or $\pi_{-}$(see Figure 12). In practical terms, this means that the factorization index of $f$ (when it exists) is always obtained explicitly, and this is all the information the [ADimKerPaired-Scalar] algorithm needs to compute the dimension of the kernel of the operators.

4.1.1. [ADimKerPaired-Scalar] examples. In this subsection we present some nontrivial examples computed with the algorithm [ADimKerPaired-Scalar]. For each pair of inputed non-singular rational functions $a, b$, defined in $\mathbb{\pi}$, the [ADimKerPaired-Scalar] algorithm gives the output (15) or (16).

All the examples were computed on a MacBook with a $1.2 \mathrm{GHz}$ Intel Core m5 processor and 8 GB of LPDDR3 RAM, running Mac OS High Sierra 10.13.6 in single user mode.

Example 4.1. Let us consider the paired singular integral operators $T_{\{a, b\}}$ and $\widetilde{T}_{\{a, b\}}$, defined in (3) and (4), with polynomial coefficients

$$
a(t)=3 t^{3}-5 t^{2}-4 \quad \text { and } \quad b(t)=t^{6}-3 t^{4}+t^{3}-2 t^{2}+2 t-1 .
$$

For

$$
f(t)=a^{-1}(t) b(t)=\frac{t^{6}-3 t^{4}+t^{3}-2 t^{2}+2 t-1}{3 t^{3}-5 t^{2}-4}
$$

the [ADimKerPaired-Scalar] algorithm computes its zeros and poles, with regard to their multiplicities, and determines whether they lie in $\mathbb{\pi}, \mathbb{\pi}_{+}$, or $\mathbb{\pi}_{-}$(see Figure 12). The factorization index is computed as $\varkappa=4-2=2$. Thus we can conclude that

$$
\operatorname{dim} \operatorname{ker} T_{\{a, b\}}=\operatorname{dim} \operatorname{ker} \widetilde{T}_{\{a, b\}}=2 .
$$

Remark 4.1. In this example, by using the [ARFact-Scalar] algorithm we get a factorization (5) of the function $a^{-1} b$, with 
and

$$
f_{+}(t)=\frac{\left(t-\alpha_{1}\right)\left(t-\alpha_{2}\right)}{3(t-2)}
$$

$$
f_{-}(t)=\frac{\left(t-\alpha_{3}\right)\left(t-\alpha_{4}\right)\left(t-\alpha_{5}\right)\left(t-\alpha_{6}\right)}{\left(t+\frac{1}{6}(1-i \sqrt{23})\right)\left(t+\frac{1}{6}(1+i \sqrt{23})\right) t^{2}},
$$

where the constants $\alpha_{i}(i=1, \ldots, 6)$ represent the exact ith root of the polynomial equation $t^{6}-3 t^{4}+$ $t^{3}-2 t^{2}+2 t-1=0$. Thus, it is possible to obtain explicitly the kernels of the paired singular integral operators $T_{\{a, b\}}$ and $\widetilde{T}_{\{a, b\}}$ using the formulas (13) and (14).

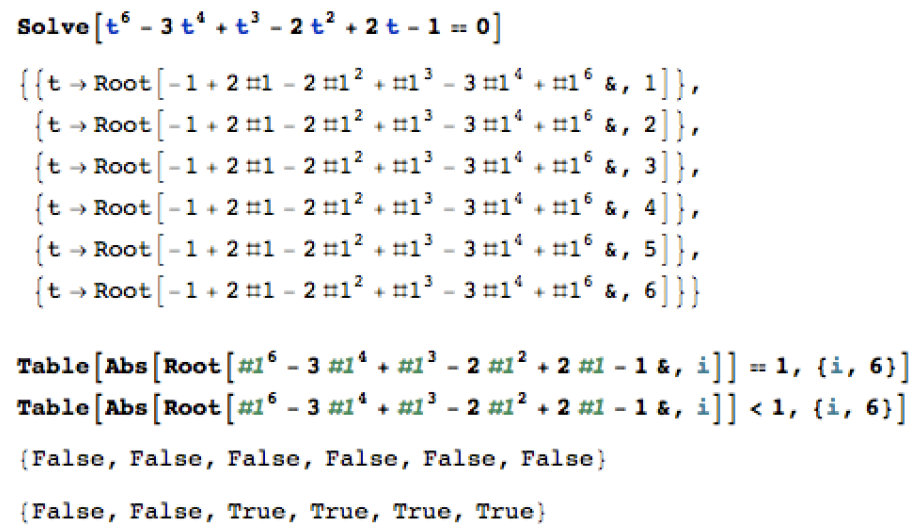

FIGURE 12. Snippet of the calculations made by the computer algebra system Mathematica for studying the zeros of the rational function $a^{-1} b$. In spite of the impossibility of computing, in an explicit way, the roots of the polynomial $t^{6}-3 t^{4}+t^{3}-2 t^{2}+2 t-1$ it is still possible to know if they lie in $\mathbb{\pi}, \mathbb{\pi}_{+}$, or $\mathbb{\pi}_{-}$. Mathematica uses the objects $\operatorname{Root}\left[\# 1^{6}-3 \# 1^{4}+\# 1^{3}-2 \# 1^{2}+2 \# 1-1 \&, i\right]$ to represent the solutions of $t^{6}-3 t^{4}+t^{3}-2 t^{2}+2 t-1=0$. In this case, there are 4 , out of 6 , poles lying inside the unit circle.

Example 4.2. Let us consider the paired singular integral operators $T_{\{a, b\}}$ and $\widetilde{T}_{\{a, b\}}$, defined in (3) and (4), with rational scalar coefficients

$$
a(t)=\left(t^{9}+5 t^{2}-1-i\right) t^{-2} \quad \text { and } \quad b(t)=t^{9}+t^{3}+2 t-10-i .
$$

For

$$
f(t)=a^{-1}(t) b(t)=\frac{t^{11}+t^{5}+2 t^{3}-(10+i) t^{2}}{t^{9}+5 t^{2}-1-i}
$$

the [ADimKerPaired-Scalar] algorithm computes its zeros and poles, with regard to their multiplicities, and determines whether they lie in $\pi, \pi_{+}$, or $\pi_{-}$. The factorization index is computed as $\varkappa=2-2=$ 0 . Thus we can conclude that

$$
\operatorname{dim} \operatorname{ker} T_{\{a, b\}}=\operatorname{dim} \operatorname{ker} \widetilde{T}_{\{a, b\}}=0 .
$$

Remark 4.2. Also for this example it is possible to obtain explicitly the kernels of the paired singular integral operators $T_{\{a, b\}}$ and $\widetilde{T}_{\{a, b\}}$ using the formulas (13) and (14).

\section{2. [AKerPaired-Scalar] algorithm}

In this subsection it is shown how the symbolic and numeric computation capabilities of Mathematica can be used to design and implement an algorithm to compute the the kernel of some classes of one-dimensional paired singular integral operators.

The [ADimKerPaired-Scalar] uses the [ARFact-Scalar] algorithm [16] and the formulas (13) and (14) to compute the kernel of one-dimensional paired singular integral operators of the form

$$
T_{\{a, b\}}=a P_{+}+b P_{-} \quad \text { and } \quad \widetilde{T}_{\{a, b\}}=P_{+} a I+P_{-} b I,
$$


defined in (3) and (4), respectively, with non-singular $a, b \in \mathcal{R}(\pi)$.

As for the [ADimKerPaired-Scalar] algorithm, the symbolic computation capabilities of Mathematica, and the pretty-print functionality, allow the [AKerPaired-Scalar] code (see Figure 13) to be very simple and syntactically similar to its analytical counterpart.

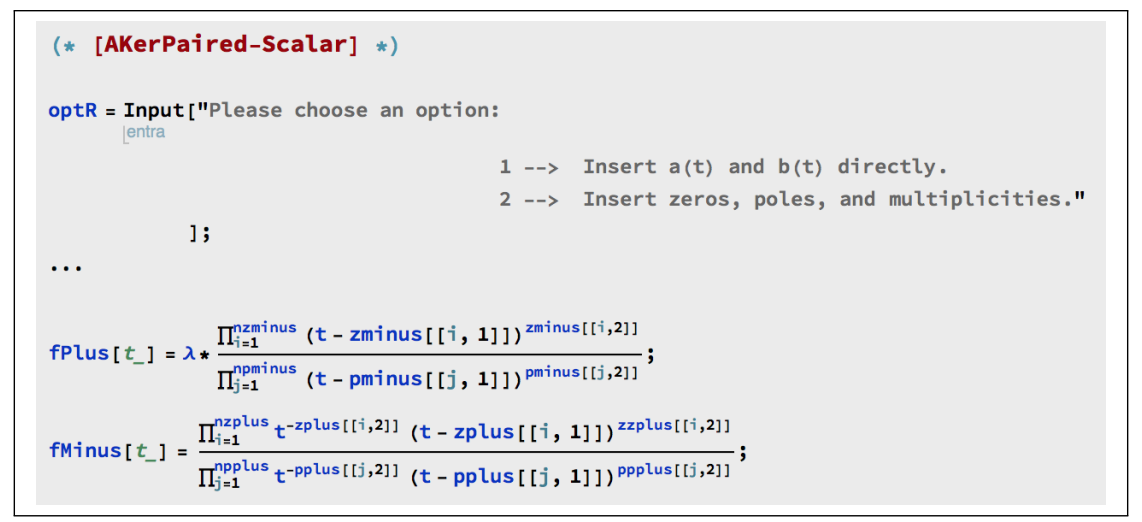

Figure 13. Snippet of the Mathematica's code responsible for the computation of a factorization of $a^{-1} b$ in the [AKerPaired-Scalar] algorithm.

There are two options to input $a(t)$ and $b(t)$ :

1. Insert $a(t)$ and $b(t)$ directly.

2. Insert zeros, poles, and multiplicities.

As for the [ADimKerPaired-Scalar] algorithm, the success of the [AKerPaired-Scalar] algorithm depends on the possibility of finding those zeros and poles by solving polynomial equations. Using the Root object it is possible to use the [ARFact-Scalar] algorithm to compute the factorization index of $f$ and the factors $f_{+}$and $f_{-}$, and this is all the information the [AKerPaired-Scalar] algorithm needs to compute the kernel of the operators.

4.2.1. [AKerPaired-Scalar] examples. In this subsection we present some examples computed with the algorithm [AKerPaired-Scalar].

The examples were computed on a MacBook with a $1.2 \mathrm{GHz}$ Intel Core m5 processor and $8 \mathrm{~GB}$ of LPDDR3 RAM, running Mac OS High Sierra 10.13.6 in single user mode.

Example 4.3. Let us consider the paired singular integral operators $T_{\{a, b\}}$ and $\widetilde{T}_{\{a, b\}}$, defined in (3) and (4), with polynomial coefficients

$$
a(t)=t-\frac{1}{2} \quad \text { and } \quad b(t)=t^{5}+2 t^{4} .
$$

The [AKerPaired-Scalar] algorithm uses the [ARFact-Scalar] algorithm to compute a factorization of the function

$$
f(t)=a^{-1}(t) b(t)=\left(t-\frac{1}{2}\right)^{-1}\left(t^{5}+2 t^{4}\right)
$$

obtaining the factorization index $\varkappa=3$ and the factors

$$
f_{+}(t)=t+2 \text { and } f_{-}(t)=t\left(t-\frac{1}{2}\right)^{-1} .
$$

Since the factorization index is bigger than zero, the output is

$$
\begin{gathered}
\operatorname{ker} T_{\{a, b\}}=\operatorname{span}\left\{\frac{\psi(t)}{t^{4}}, \frac{\psi(t)}{t^{3}}, \frac{\psi(t)}{t^{2}}\right\} \text {, where } \psi(t)=t^{5}+2 t^{4}-t+\frac{1}{2} \\
\operatorname{ker} \widetilde{T}_{\{a, b\}}=\operatorname{span}\left\{\frac{1}{t^{4}}, \frac{1}{t^{3}}, \frac{1}{t^{2}}\right\} .
\end{gathered}
$$


Example 4.4. Let us consider the paired singular integral operators $T_{\{a, b\}}$ and $\widetilde{T}_{\{a, b\}}$, defined in (3) and (4), with polynomial coefficients

$$
a(t)=t^{21}+t^{13}-4 t^{2} \quad \text { and } \quad b(t)=t^{10}-2 t^{2}+t-1 .
$$

The [AKerPaired-Scalar $]$ algorithm uses the [ARFact-Scalar $]$ algorithm to compute a factorization of the function

$$
f(t)=a^{-1}(t) b(t)=\left(t^{21}+t^{13}-4 t^{2}\right)^{-1}\left(t^{10}-2 t^{2}+t-1\right)
$$

obtaining the factorization index $\varkappa=0$. So, the output is that the operators have trivial kernels,

$$
\operatorname{ker} T_{\{a, b\}}=\operatorname{ker} \widetilde{T}_{\{a, b\}}=\{0\} .
$$

Remark 4.3. This case shows that, since the factorization index of $f$ can always be obtained explicitly, when $\varkappa \leqslant 0$, the [AKerPaired-Scalar] have all the information to give the output of trivial kernels (even for rational functions containing polynomials with high degree).

\section{3. [ADimKerPaired-Matrix] algorithm}

In this subsection it is explained how the symbolic and numeric computation capabilities of the computer algebra system Mathematica can be used to design an algorithm to compute the dimension of the kernel of paired singular integral operators of the form

$$
T_{\{a, b\}}=a P_{+}+b P_{-} \quad \text { and } \quad \widetilde{T}_{\{a, b\}}=P_{+} a I+P_{-} b I,
$$

defined in (3) and (4), respectively, with non-singular $a, b \in[\mathcal{R}(\mathbb{\pi})]_{n, n}, n \geqslant 2$.

Due to the symbolic and numeric capabilities of Mathematica several nontrivial examples such that

$$
\operatorname{dim} \operatorname{ker} T_{\{a, b\}} \neq \operatorname{dim} \operatorname{ker} \widetilde{T}_{\{a, b\}}
$$

can be easily constructed (see Example 4.5 and Example 4.6 .

In the design of this spectral algorithm we used the factorization algorithm [ARFact-Matrix] [16], that computes explicit factorizations for given factorable rational matrix function defined on the unit circle. For this reason, the success of the [ADimKerPaired-Matrix] algorithm depends on the possibility of finding solutions of polynomial equations. However, due to the complexity of the matrix case, it is not as feasible as before to use the Root objects to obtain an explicit matrix function factorization when working with polynomials of a high degree. In fact, one crucial step of this algorithm is finding the zeros of the determinant of a rational matrix function defined through the matrix functions $a$ and $b$. This means that the dimension of the matrix is also a limiting factor, even when its entries are rational functions with low degree polynomials. We also note that, although the final factorization may have relatively simple entries, if we were to use the traditional pencil and paper tools the intermediate calculations would take typically many working hours, up to the point of infeasibility, even for low matrix orders.

The [ADimKerPaired-Matrix] algorithm can be applied to a given paired singular integral operator of classes (3) and (4), with non-singular $a, b \in[\mathcal{R}(\pi)]_{n, n}, n \geqslant 2$.

As in the case of the [ADimKerPaired-Scalar] algorithm, for the [ADimKerPaired-Matrix] algorithm there are two options to input the entries of $a(t)$ and $b(t)$ :

1. Insert the entries of $a(t)$ and $b(t)$ directly.

2. Insert, for each entry, the numerator and the poles (and its multiplicities).

Due to the non-commutativity of matrix function multiplication and the usage of the [ARFactMatrix] algorithm, the code of [ADimKerPaired-Matrix] must be significantly more complex than the code of the [ADimKerPaired-Scalar] algorithm.

Figure 14 presents the flowchart of the [ADimKerPaired-Matrix] algorithm ${ }^{14}$ (considering a nonsingular matrix function $a$ ).

${ }^{14}$ If $b^{-1} \in[\mathcal{R}(\mathbb{T})]_{n, n}$, then a similar algorithm can be described using the matrix functions $b^{-1} a$ and $a b^{-1}$. 


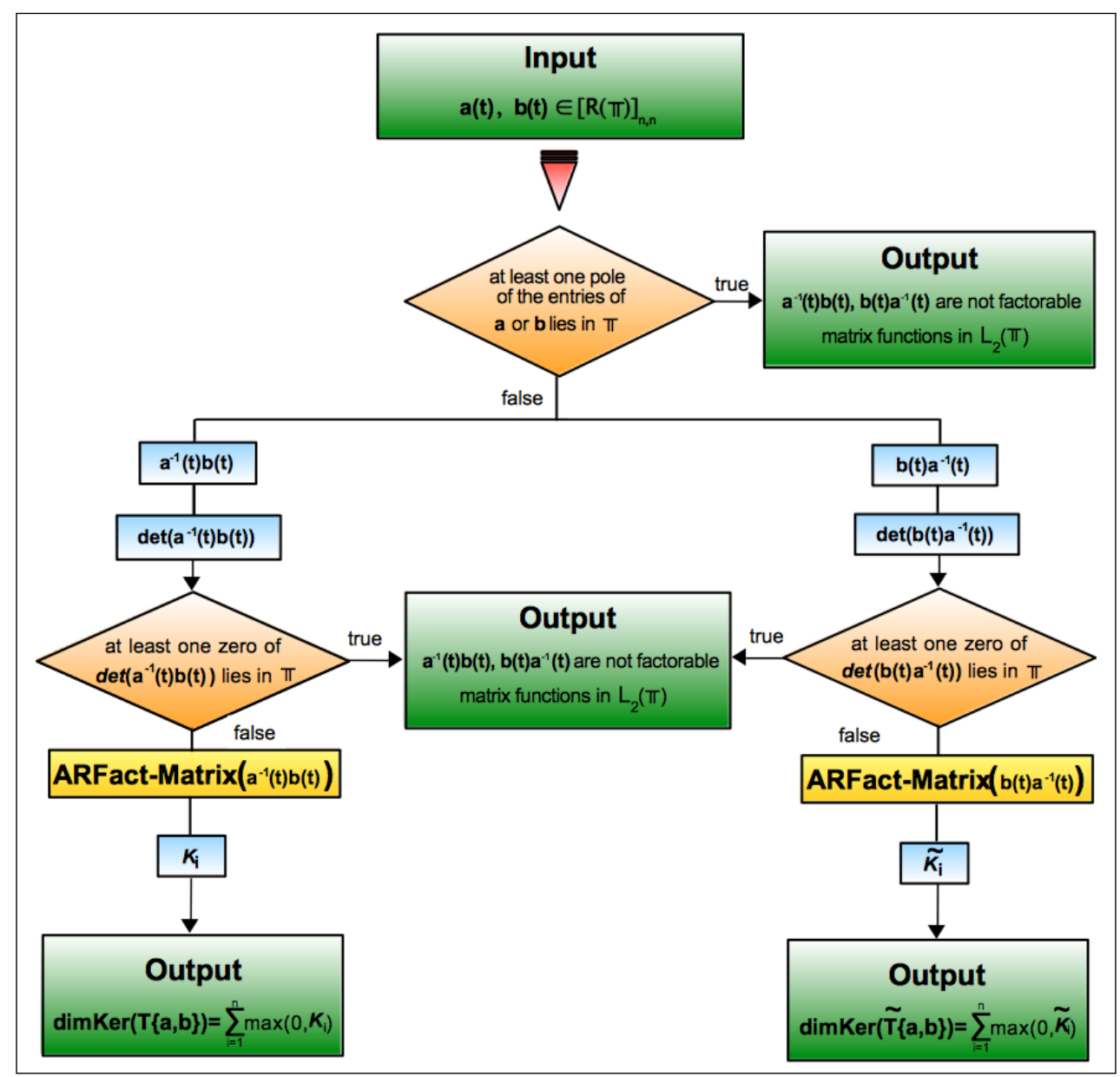

Figure 14. Flowchart of the [ADimKerPaired-Matrix] algorithm.

4.3.1. [ADimKerPaired-Matrix] examples. In this subsection we present some nontrivial examples computed with the algorithm [ADimKerPaired-Matrix]. For each pair of inputed non-singular rational matrix functions $a, b$, defined in $\mathbb{\pi}$, the [ADimKerPaired-Scalar] algorithm computes the dimension of the kernel of the operators $T_{\{a, b\}}$ and $\widetilde{T}_{\{a, b\}}$.

All the examples were computed on a MacBook with a $1.2 \mathrm{GHz}$ Intel Core m5 processor and 8 GB of LPDDR3 RAM, running Mac OS High Sierra 10.13.6 in single user mode.

Example 4.5. Let us consider the paired singular integral operators $T_{\{a, b\}}$ and $\widetilde{T}_{\{a, b\}}$, defined in (3) and (4), with rational matrix coefficients

$$
a(t)=\left(\begin{array}{cc}
t^{-1} & -i \\
-2 t^{-1} & t+2 i
\end{array}\right) \quad \text { and } \quad b(t)=\left(\begin{array}{cc}
-i & t^{-1} \\
t+2 i & -2 t^{-1}
\end{array}\right) .
$$

There are no poles in the entries of the matrix functions $a$ and $b$ lying in $\pi$. In this case $a b \neq b a$. As a consequence, the equality (8) is not necessarily satisfied. The [ADimKerPaired-Matrix] algorithm constructs the matrix function

$$
a^{-1}(t) b(t)=\left(\begin{array}{cc}
0 & 1 \\
1 & 0
\end{array}\right)
$$


Taking into account that $\operatorname{det}\left(a^{-1} b\right)$ has no zeros in $\pi$ the [ADimKerPaired-Matrix] algorithm constructs the matrix function

$$
b(t) a^{-1}(t)=\left(\begin{array}{cc}
\left(-i t^{3}+2 t^{2}+2\right) t^{-2} & t^{-2}+1 \\
\left(t^{4}+4 i t^{3}-4 t^{2}-4\right) t^{-2} & \left(i t^{3}-2 t^{2}-2\right) t^{-2}
\end{array}\right)
$$

Then uses the [ARFact-Matrix] algorithm to compute the left partial indices of the matrix function $a^{-1} b, \varkappa_{1}=\varkappa_{2}=0$, and the left partial indices of the matrix function $b a^{-1}, \tilde{\varkappa}_{1}=1$ and $\tilde{\varkappa}_{2}=-1$.

The Output is

$$
\operatorname{dim} \operatorname{ker} T_{\{a, b\}}=0 \text { and } \operatorname{dim} \operatorname{ker} \widetilde{T}_{\{a, b\}}=1 .
$$

Example 4.6. Let us consider the paired singular integral operators $T_{\{a, b\}}$ and $\widetilde{T}_{\{a, b\}}$, defined in (3) and (4), with rational matrix coefficients

$$
a(t)=\left(\begin{array}{ccc}
0 & 0 & t \\
0 & t^{-1} & 0 \\
1 & 0 & 0
\end{array}\right) \quad \text { and } b(t)=\left(\begin{array}{ccc}
1 & 0 & 0 \\
0 & t^{-1} & 0 \\
0 & 0 & t
\end{array}\right) .
$$

There are no poles in the entries of the matrix functions $a$ and $b$ lying in $\pi$. In this case $a b \neq b a$. As a consequence, the equality (8) is not necessarily satisfied. The [ADimKerPaired-Matrix] algorithm constructs the matrix function

$$
a^{-1}(t) b(t)=\left(\begin{array}{ccc}
0 & 0 & t \\
0 & 1 & 0 \\
t^{-1} & 0 & 0
\end{array}\right)
$$

and computes (with the [ARFact-Matrix] algorithm) a left factorization with the left partial indices $\varkappa_{1}=1, \varkappa_{2}=0, \varkappa_{3}=-1$. On the other hand, the matrix function

$$
b(t) a^{-1}(t)=\left(\begin{array}{ccc}
0 & 0 & 1 \\
0 & 1 & 0 \\
1 & 0 & 0
\end{array}\right)
$$

admits a left factorization with the left partial indices $\varkappa_{1}=\varkappa_{2}=\varkappa_{3}=0$.

So, due to Theorem 3.2 and Theorem 3.3 the Output is

$$
\operatorname{dim} \operatorname{ker} T_{\{a, b\}}=1 \text { and } \operatorname{dim} \operatorname{ker} \widetilde{T}_{\{a, b\}}=0 .
$$

Examples 4.5 and 4.6 illustrate the fact that the dimensions of the kernels of $T_{\{a, b\}}$ and $\widetilde{T}_{\{a, b\}}$ are not necessarily the same. Furthermore, these examples exemplify that when the operators $P_{+}+$ $(\operatorname{det} f) P_{-}$and $P_{+}+(\operatorname{det} g) P_{-}$have trivial kernels the operators $T_{\{a, b\}}$ and $\widetilde{T}_{\{a, b\}}$ can have trivial or nontrivial kernels (see Remark 3.5).

Example 4.7. Let us consider the paired singular integral operators $T_{\{a, b\}}$ and $\widetilde{T}_{\{a, b\}}$, defined in (3) and (4), with rational matrix coefficients

$$
a(t)=\left(\begin{array}{ccccccc}
\frac{i}{t-3 i} & 0 & 0 & 0 & 0 & 0 & 0 \\
0 & \frac{1}{5 t-1} & 0 & 0 & 0 & 0 & 0 \\
-\frac{1}{i t+3} & 0 & \frac{t-3 i}{i t-+3} & 0 & 0 & 0 & 0 \\
0 & 0 & 0 & 1 & 0 & 0 & 0 \\
0 & 0 & 0 & 0 & 0 & \frac{1}{t} & 0 \\
0 & 0 & 0 & 0 & 1 & 0 & 0 \\
-\frac{i}{i t+3} & 0 & 0 & 0 & 0 & 0 & 1
\end{array}\right)
$$

and 


$$
b(t)=\left(\begin{array}{ccccccc}
0 & \frac{i}{t-6} & 0 & \frac{1}{t-4} & 0 & 0 & \frac{1}{t-5} \\
0 & \frac{2 i}{5 t-1} & \frac{t+1}{5 t-1} & 0 & 1 & 0 & 0 \\
0 & -\frac{1}{t-6} & 0 & \frac{i(t-1)}{t-2} & 0 & 0 & \frac{i}{t-2} \\
0 & 0 & -1 & 0 & 1 & 0 & 0 \\
1 & 0 & 0 & 1 & 1 & 0 & 0 \\
1 & \frac{1}{t} & 0 & 0 & 0 & 0 & 0 \\
0 & -\frac{i}{t(t-6)} & 0 & -\frac{1}{t(t-4)} & 0 & 1 & \frac{-1}{t(t-4)}
\end{array}\right) .
$$

There are no poles in the entries of the matrix functions $a$ and $b$ lying in $\mathbb{\pi}$. In this case $a b \neq b a$. As a consequence, the equality (8) is not necessarily satisfied.

The [ADimKerPaired-Matrix] algorithm constructs the matrix function

$$
g(t)=b(t) a^{-1}(t)=\left(\begin{array}{ccccccc}
\frac{1}{t-5} & \frac{i(5 t-1)}{t-6} & 0 & \frac{1}{t-4} & 0 & 0 & \frac{1}{t-5} \\
\frac{t+1}{5 t-1} & 2 i & \frac{i(t+1)}{5 t-1} & 0 & 0 & 1 & 0 \\
\frac{i}{t-2} & -\frac{5 t-1}{t-6} & 0 & \frac{i(t-1)}{t-2} & 0 & 0 & \frac{i}{t-2} \\
-1 & 0 & -i & 0 & 0 & 1 & 0 \\
-3 i+t & 0 & 0 & 1 & 0 & 1 & 0 \\
-3 i+t & \frac{5 t-1}{t} & 0 & 0 & 0 & 0 & 0 \\
-\frac{1}{(t-4) t} & -\frac{i(5 t-1)}{(t-6) t} & 0 & -\frac{1}{(t-4) t} & t & 0 & -\frac{1}{(t-4) t}
\end{array}\right)
$$

and computes (using the [ARFact-Matrix] algorithm) a left factorization with the left partial indices $\varkappa_{1}=1$ and $\varkappa_{i}=0, \forall i=\overline{2,6}$ (see Figure 15$)$. So, $\operatorname{dim} \operatorname{ker}\left(P_{+}+(\operatorname{det} g) P_{-}\right)=1$ and $\left.\operatorname{dim} \operatorname{ker} \widetilde{T}_{\{a, b\}}\right)=1$.

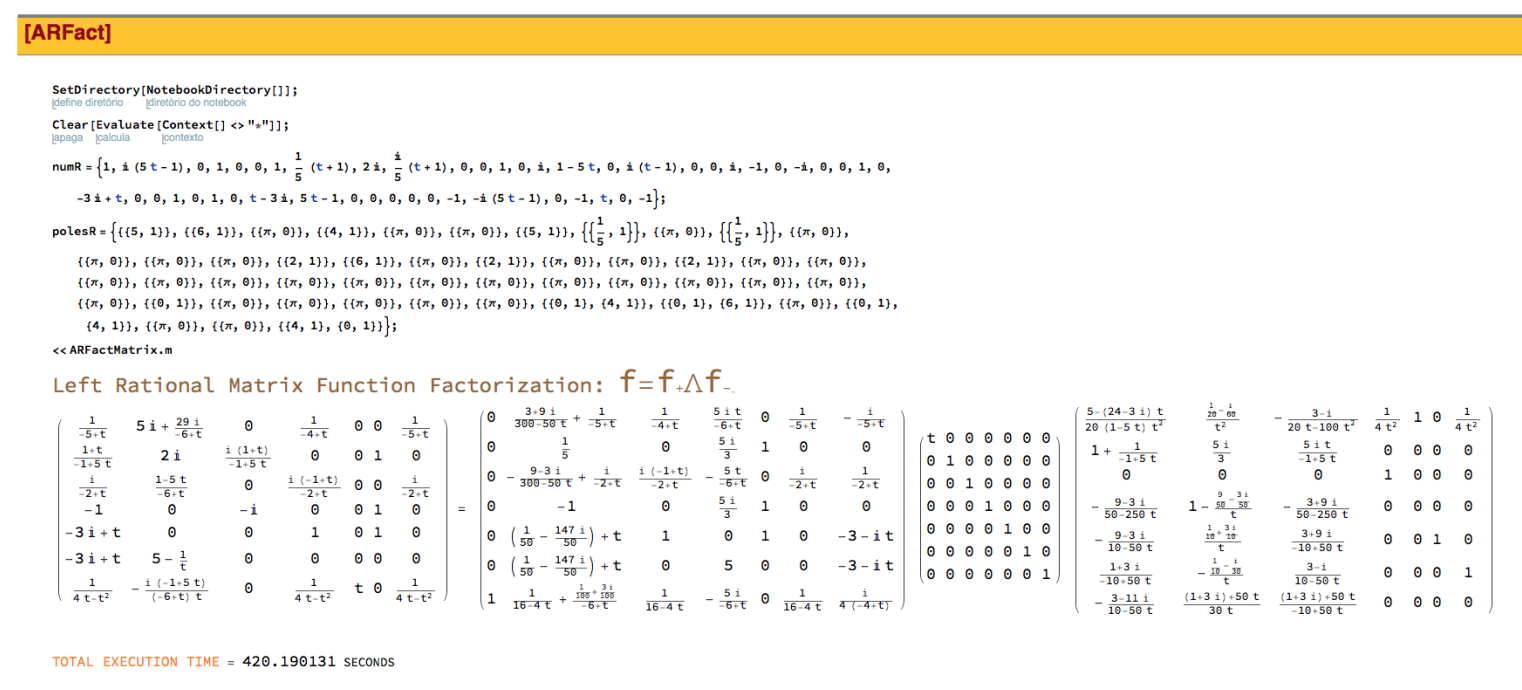

FIGURE 15. Left factorization computed with [ARFact-Matrix] algorithm.

In this example, the [ARFact-Matrix] algorithm, due the dimension of the matrix function, is unable to determine the left partial indices of the matrix function $f=a^{-1} b$. However, since $\operatorname{dim} \operatorname{ker}\left(P_{+}+(\operatorname{det} f) P_{-}\right)=1>0$, through the Theorem 3.9 we can conclude that $\left.\operatorname{dim} \operatorname{ker} T_{\{a, b\}}\right)>0$.

Example 4.8. Let us consider the paired singular integral operators $T_{\{a, b\}}$ and $\widetilde{T}_{\{a, b\}}$, defined in (3) and (4), with rational matrix coefficients 
and

$$
a(t)=\left(\begin{array}{ccccccc}
\frac{1}{t-2} & 0 & 0 & 0 & 0 & 0 & 0 \\
0 & \frac{1}{4 t-1} & 0 & 0 & 0 & 0 & 0 \\
\frac{i}{t-2} & 0 & -i & 0 & 0 & 0 & 0 \\
0 & 0 & 0 & 1 & 0 & 0 & 0 \\
0 & 0 & 0 & 0 & 0 & \frac{1}{t} & 0 \\
0 & 0 & 0 & 0 & 1 & 0 & 0 \\
-\frac{1}{t(t-2)} & 0 & 0 & 0 & 0 & 0 & 1
\end{array}\right)
$$

$$
b(t)=\left(\begin{array}{ccccccc}
0 & \frac{i}{t-7 i} & 0 & \frac{1}{t-2} & 0 & 0 & \frac{1}{t-2} \\
0 & \frac{2 i}{4 t-1} & \frac{t+1}{4 t-1} & 0 & \frac{1}{t} & 0 & 0 \\
0 & -\frac{1}{t-7 i} & 0 & \frac{i(t-1)}{t-2} & 0 & 0 & \frac{i}{t-2} \\
0 & 0 & -1 & 0 & 1 & 0 & 0 \\
\frac{1}{t} & 0 & 0 & 1 & \frac{1}{t^{2}} & 0 & 0 \\
1 & \frac{1}{t} & 0 & 0 & 0 & 0 & 0 \\
0 & -\frac{i}{t(t-7 i)} & 0 & -\frac{1}{t(t-2)} & 0 & 1 & \frac{-1}{t(t-2)}
\end{array}\right) .
$$

There are no poles in the entries of the matrix functions $a$ and $b$ lying in $\pi$. In this case $a b \neq b a$. As a consequence, the equality (8) is not necessarily satisfied.

The [ADimKerPaired-Matrix] algorithm constructs the matrix function

$$
f(t)=a^{-1}(t) b(t)=\left(\begin{array}{ccccccc}
0 & \frac{i(-2+t)}{-7 i+t} & 0 & 1 & 0 & 0 & 1 \\
0 & 2 i & 1+t & 0 & \frac{4 t-1}{t} & 0 & 0 \\
0 & 0 & 0 & -1 & 0 & 0 & 0 \\
0 & 0 & -1 & 0 & 1 & 0 & 0 \\
1 & \frac{1}{t} & 0 & 0 & 0 & 0 & 0 \\
1 & 0 & 0 & t & \frac{1}{t} & 0 & 0 \\
0 & 0 & 0 & 0 & 0 & 1 & 0
\end{array}\right),
$$

and computes $\operatorname{det}\left(a^{-1}(t) b(t)\right)=\left(t^{2}+(5+2 i) t-1\right) t^{-2}$. There are no zeros of the determinant of $a^{-1}(t) b(t)$ in $\mathbb{\pi}$. The [ARFact-Matrix] algorithm computes the left partial indices $\varkappa_{i}$ of $f$ as $\varkappa_{1}=$ $\varkappa_{2}=\varkappa_{3}=\varkappa_{4}=\varkappa_{5}=\varkappa_{6}=0$ and $\varkappa_{7}=-1$ (see Appendix). From Theorem 3.2 we can conclude that

$$
\operatorname{dim} \operatorname{ker} T_{\{a, b\}}=0 .
$$

In this example the [ARFact-Matrix] algorithm failed to determine the left partial indices of the matrix function $b a^{-1}$ (the function $f$ of the Example 3.3) relative to the operator $\widetilde{T}_{\{a, b\}}$ due to the high degree of the polynomials of the entries of the matrix function $b a^{-1}$. Since $\operatorname{dim} \operatorname{ker}\left(P_{+}+(\operatorname{det} g) P_{-}\right)=0$ it is not possible to apply Theorem 3.9.

\section{Special classes of paired singular integral operators}

This section is devoted to special classes of paired singular integral operators with essentially bounded coefficients, defined on the unit circle. Due the specificity and complexity of these operators it is very hard to automate the exploration process. However, it is still possible to use, in a step-by-step manner, the symbolic computation capabilities of Mathematica, to explore the corresponding kernel for some classes of such paired singular integral operators.

\subsection{On the factorability of essentially bounded hermitian matrix functions}

Let us consider essentially bounded matrix functions of the form

$$
A_{\gamma}(\varphi)=\left(\begin{array}{cc}
e & \varphi \\
\varphi^{*} & \varphi^{*} \varphi+\gamma e
\end{array}\right)
$$

where $\varphi$ is an essentially bounded matrix function on the unit circle, that is, $\varphi \in\left[L_{\infty}(\pi)\right]_{n, n}, \varphi^{*}$ is the hermitian adjoint of $\varphi$, and $\gamma \in \mathbb{C}$. 
In $[11,14,16,17]$ we present necessary and sufficient conditions for the existence of a canonical generalized factorization of $A_{\gamma}(\varphi)$. Although we have theoretical results for $\varphi \in\left[L_{\infty}(\pi)\right]_{n, n}$, we can always assume, without loss of generality, that $\varphi \in\left[L_{\infty}^{+}(\pi)\right]_{n, n}$, that is, $\varphi$ is an essentially bounded matrix function, holomorphic and bounded on $\mathbb{\pi}_{+}$(see, for instance, $\left.[14,17,18,19]\right)$.

For the particular second-order case, it is possible to show that the study of the factorability of essentially bounded hermitian matrix functions, with negative determinant and definite diagonal elements, can be reduced [11, 17, 42] to the study of the factorability of matrix functions of the class (18). In addition, a canonical generalized factorization of matrix functions of the type (18) has applications in several scientific research areas [1, 15, 18, 21, 23, 27, 39, 41, 42].

The [AFact] algorithm [17] computes explicit factorizations for matrix functions of some subclasses of class (18) for $\varphi \in H_{r, \theta}$.

\subsection{On the kernel of special classes of paired singular integral operators}

Let us now consider the special classes of paired singular integral operators of the form

$$
T_{\gamma\{a, b\}}=a P_{+}+b P_{-},
$$

defined in (3) with $a, b \in\left[L_{\infty}(\pi)\right]_{2 n, 2 n}$ such that ${ }^{15} a^{-1} b$ belongs to class (18), for a matrix function $\varphi \in\left[L_{\infty}(\pi)\right]_{n, n}$ and $\gamma \in \mathbb{C}$.

Let us also consider the special classes of paired singular integral operators of the form

$$
\widetilde{T}_{\gamma\{a, b\}}=P_{+} a I+P_{-} b I
$$

defined in (4) with $a, b \in\left[L_{\infty}(\pi)\right]_{2 n, 2 n}$ such that ${ }^{16} b a^{-1}$ belongs to class (18), for a matrix function $\varphi \in\left[L_{\infty}(\pi)\right]_{n, n}$ and $\gamma \in \mathbb{C}$.

In [24], based directly on the ideas and concepts that were presented in [17], we formulated new results that relate the spectra of operators (19) and (20) with the spectra of the special class of self-adjoint singular integral operators

where $\varphi \in\left[L_{\infty}(\pi)\right]_{n, n}$.

$$
P_{+} \varphi P_{-} \varphi^{*} P_{+}
$$

Now, we formulate new results that relate the kernel of operators (19) and (20) with the special class of self-adjoint Hankel operators

where $\varphi \in\left[L_{\infty}(\pi)\right]_{n, n}$.

$$
P_{-} \varphi^{*} P_{+} \varphi P_{-}
$$

Theorem 5.1. Let $T_{\gamma\{a, b\}}$ be a singular integral operator of class (19), for a matrix function $\varphi \in$ $\left[L_{\infty}(\pi)\right]_{n, n}$ and $\gamma \in \mathbb{C}$. Then

$$
\operatorname{dim} \operatorname{ker} T_{\gamma\{a, b\}}=\operatorname{dim} \operatorname{ker}\left(P_{-} \varphi^{*} P_{+} \varphi P_{-}+\gamma I\right) .
$$

Proof. If $\gamma \in \mathbb{C} \backslash \mathbb{R}_{0}^{-}$, by Corollary 2.2 of [17] we can conclude that the matrix function $a^{-1} b$ admits a left canonical factorization in $L_{2}(\pi)$. As a consequence, $\operatorname{dim} \operatorname{ker}\left(P_{-} \varphi^{*} P_{+} \varphi P_{-}+\gamma I\right)=0$ and $\operatorname{dim} \operatorname{ker} T_{\gamma\{a, b\}}=0$.

If $\gamma<0$, then the dimension of the kernel of the Hankel operator $P_{-} \varphi^{*} P_{+} \varphi P_{-}+\gamma I$ corresponds [17] to the sum of the positive left partial indices of the hermitian matrix function $a^{-1} b$, that is, the dimension of the kernel of $T_{\gamma\{a, b\}}$.

Based on Theorem 3.2 of [23] the following result can be formulated.

Theorem 5.2. If $P_{-} \varphi=\varphi$ and $\gamma \in \mathbb{C} \backslash\{0\}$, then $T_{\gamma\{a, b\}}$ has a trivial kernel.

Using Theorem 3.3 of [23] we get the following result.

Theorem 5.3. If $\gamma \in \mathbb{C} \backslash \mathbb{R}_{0}^{-}$, then $T_{\gamma\{a, b\}}$ has a trivial kernel.

\footnotetext{
${ }_{15}$ The same idea can be applied when $b^{-1} a$ belongs to class (18) and admits a right generalized factorization in $L_{2}(\pi)$.

${ }^{16}$ The same idea can be applied when $a b^{-1}$ belongs to class (18) and admits a right generalized factorization in $L_{2}(\pi)$.
} 
Remark 5.1. Similar results ${ }^{17}$ to Theorems 5.1, 5.2, and 5.3 were obtained for the kernel of singular integral operators $\widetilde{T}_{\gamma\{a, b\}}$, defined in (20).

For the case when $\varphi \in H_{r, \theta}$, we designed the generalized factorization algorithm [AFact] [11, 17] that computes a left generalized factorization of factorable essentially bounded second-order matrix functions of type (18), for any general inner function $\theta$. In particular, the [AFact] algorithm allows us to know if a matrix function of the class (18) admits, or not, a left generalized factorization (5). Moreover, if $A_{\gamma}(\varphi)$ is factorable, the algorithm allows us to determine if the generalized factorization is canonical or non-canonical, and it gives us an explicit left generalized factorization of the matrix function. Note that the computations of the [AFact] algorithm do not depend on the degree of the polynomials that may eventually be part of inner function $\theta$. Therefore, for some subclasses of operators (19) and (20), whose kernels cannot be studied with the [ARFact-Matrix] algorithm due to the many zeros and poles present in the entries of the corresponding matrix coefficients, it may still be possible to use [AFact] to perform this analysis (see Example 5.1). In addition, our [SInt] algorithm [15] computes the singular integral $S_{\top} \varphi$ and its projections $P_{ \pm} \varphi$ for an essentially bounded function $\varphi$, and can be used to check if $P_{-} \varphi=\varphi$ (see Example 5.1).

5.2.1. Essentially bounded examples. Let us now show how the symbolic and numeric computation capabilities of the computer algebra system Mathematica (by using the algorithms [SInt] and [AFact]) can be used to construct some interesting examples for these special classes of paired singular integral operators.

Example 5.1. We consider the paired singular integral operators $T_{\gamma\{a, b\}}$ and $\widetilde{T}_{\gamma\{a, b\}}$ defined in (19) and (20) with essentially bounded matrix coefficients

$$
a=\left(\begin{array}{cc}
1 & 0 \\
-\bar{\varphi} \gamma^{-1} & \gamma^{-1}
\end{array}\right) \quad \text { and } \quad b=\left(\begin{array}{cc}
1 & \varphi \\
0 & 1
\end{array}\right),
$$

where $\varphi \in L_{\infty}(\pi), \gamma \in \mathbb{C} \backslash\{0\}$, and the overline in $\bar{\varphi}$ denotes the complex conjugate of $\varphi$ in the unit circle.

Let us note that in this case $a b \neq b a$. Therefore, the equality (8) is not necessarily satisfied.

Paired singular integral operator $T_{\gamma\{a, b\}}$ : Let us now study the dimension of the kernel of the paired singular integral operator (19).

Since

$$
a^{-1} b=\left(\begin{array}{cc}
1 & \varphi \\
\bar{\varphi} & |\varphi|^{2}+\gamma
\end{array}\right)
$$

belongs to the class (18), the Theorems 5.1, 5.2, and 5.3 can be used to study the kernel of the operator (19).

Case 1. Let us consider $\varphi(t)$ represented as $\varphi(t)=x(t) y_{-}(t)$, where $\overline{y_{-}}$is an arbitrary essentially bounded function on the unit circle, analytic in the interior of the unit circle, $x$ is a rational function without poles on $\mathbb{\pi}$, and $\gamma$ is an arbitrary non-null complex value. In this case the [SInt] algorithm [15] can be used to study the kernel of operator (19).

For instance, if

$$
x(t)=\frac{(3+i) t^{4}-(9-\sqrt{7} i) t+1}{(i-10) t^{9}+2 t^{7}+t^{6}+1}
$$

the calculation technique [SInt] concludes that $S_{\pi} \varphi=-\varphi$, that is, $P_{-} \varphi=\varphi$. Therefore, by Theorem 5.2

$$
\operatorname{dim} \operatorname{ker}\left(T_{\gamma\{a, b\}}\right)=0 .
$$

Furthermore, if $y_{-}$is a rational function, the study of the kernel of operator (19) maybe it could also be done by the [ARFact-Matrix] algorithm. However, due to the complexity of the matrix case, it

\footnotetext{
${ }^{17}$ Considering a left generalized factorization (5) of the matrix function $b a^{-1}$ or a right generalized factorization (5) of the matrix function $a b^{-1}$.
} 
is not as feasible as before to use the Root objects to obtain an explicit matrix function factorization when working with polynomials of such a high degree.

Case 2. Let $\varphi$ be an arbitrary essentially bounded function and $\gamma \in \mathbb{C} \backslash \mathbb{R}_{0}^{-}$. By Theorem 5.3 we can conclude immediately, without using the symbolic computation capabilities of Mathematica, that

$$
\operatorname{dim} \operatorname{ker}\left(T_{\gamma\{a, b\}}\right)=0 .
$$

Case 3. Let us consider $\varphi(t)=\theta(t)(t-2)^{-1}$, where $\theta$ is an arbitrary inner function and $\gamma<0$. In this case the [AFact] algorithm can be used to study the kernel of operator (19).

(i) Let $\theta$ be a differentiable inner function in a neighborhood of $t=1$, in $\pi$, and $\gamma=-1$. The factorization algorithm [AFact] gives the output that the matrix function $a^{-1} b$ admits a left canonical generalized factorization in $L_{2}(\pi)$. As such, we can conclude by Theorem 3.5 that

$$
\operatorname{dim} \operatorname{ker}\left(T_{\gamma\{a, b\}}\right)=0 .
$$

(ii) Let $\theta$ be a differentiable inner function in a neighborhood of $t=-1$, in $\pi$, such that satisfies the condition ${ }^{18} \theta^{\prime}(-1)=0$ and $\gamma=-\frac{1}{9}$. The [AFact] algorithm gives the output that the left partial indices of the matrix function $a^{-1} b$ are $\varkappa_{1}=1$ and $\varkappa_{2}=-1$. As such, we can conclude by Theorem 3.5 that

$$
\operatorname{dim} \operatorname{ker}\left(T_{\gamma\{a, b\}}\right)=1 .
$$

Paired singular integral operator $\widetilde{T}_{\gamma\{a, b\}}$ : Let us now study the dimension of the kernel of the paired singular integral operator (20).

Since

$$
b a^{-1}=\left(\begin{array}{cc}
1+|\varphi|^{2} & \gamma \varphi \\
\bar{\varphi} & \gamma
\end{array}\right)
$$

that is,

$$
b a^{-1}=\left(\begin{array}{ll}
0 & 1 \\
1 & 0
\end{array}\right)\left(\begin{array}{cc}
1 & \bar{\varphi} \\
\varphi & 1+|\varphi|^{2}
\end{array}\right)\left(\begin{array}{ll}
0 & \gamma \\
1 & 0
\end{array}\right)
$$

the study of the factorability of this matrix function can be reduced to the study of the factorability of

$$
\left(\begin{array}{cc}
1 & \bar{\varphi} \\
\varphi & 1+|\varphi|^{2}
\end{array}\right)
$$

which is a matrix function of class (18). Corollary 2.2 and Remark 2.4 of [17] can be used to conclude immediately, without the need to use the symbolic computation capabilities of Mathematica, that

$$
\operatorname{dim} \operatorname{ker}\left(\widetilde{T}_{\gamma\{a, b\}}\right)=0,
$$

for all $\varphi \in L_{\infty}(\mathbb{\pi})$ and $\gamma \in \mathbb{C} \backslash\{0\}$.

\section{CONCLUSIONS}

The design of our analytical algorithms is focused on the possibility of implementing on a computer all, or a significant part, of the extensive symbolic and numeric calculations present in the algorithms. The methods developed rely on innovative techniques of operator theory and have a great potential of extension to ever more complex and general problems. Also, by implementing these methods on a computer, new and powerful tools are created for exploring that same potential, making the results of lengthy and complex calculations available in a simple way to researchers of different areas.

- We are considering the design and implementation of other factorization, spectral and kernel algorithms. In particular, we hope to publish in the near future some results concerning algorithms to explore the spectra and compute the kernels of singular integral operators related with Hankel and commutator operators.

\footnotetext{
${ }^{18}$ This condition is provided explicitly in the output of the [AFact] algorithm. It arises from the construction of an homogeneous linear system which we know to be uniquely solvable when $-\gamma \in \sigma\left(P_{-} \bar{\varphi} P_{+} \varphi P_{-}\right)$.
} 
- Furthermore, we note that the majority of the concepts and results established for the unit circle within operator theory can be generalized for the real line. It is our opinion that the design and implementation of analytical algorithms that work with singular integral operators defined on the real line can constitute a very interesting new line of research.

- We hope that our work within the operator theory, and with Mathematica, will help in the path to the future design and implementation of several other analytical algorithms, with numerous applications in many areas of research and technology.

- We also hope that, going forward, these analytical methods, and their implementation using a computer algebra system with large symbolic and numeric computation capabilities, may contribute to the numerical approach in operator theory.

\section{APPENDIX}

\section{Example 4.8}

\section{Left non-canonical factorization of $f$ :}

$$
\begin{aligned}
& f_{+}(t)=\left(\begin{array}{ccccccc}
i-\frac{28-98 i}{k_{1}(t-7 i)} & \frac{-2(7+2 i) k_{2} t}{k_{1}(t-7 i)} & 0 & 1 & 0 & 1 & 0 \\
k_{3} & -1-k_{2} t & 1+t & 0 & 0 & 0 & -1+4 t \\
0 & 0 & 0 & -1 & 0 & 0 & 0 \\
1 & 0 & -1 & 0 & 0 & 0 & t \\
0 & 1 & 0 & 0 & 0 & 0 & 0 \\
0 & 1 & 0 & t & 0 & 0 & 1 \\
0 & 0 & 0 & 0 & 1 & 0 & 0
\end{array}\right), \\
& \Lambda(t)=\left(\begin{array}{ccccccc}
1 & 0 & 0 & 0 & 0 & 0 & 0 \\
0 & 1 & 0 & 0 & 0 & 0 & 0 \\
0 & 0 & 1 & 0 & 0 & 0 & 0 \\
0 & 0 & 0 & 1 & 0 & 0 & 0 \\
0 & 0 & 0 & 0 & 1 & 0 & 0 \\
0 & 0 & 0 & 0 & 0 & 1 & 0 \\
0 & 0 & 0 & 0 & 0 & 0 & \frac{1}{t}
\end{array}\right), f_{-}(t)=\left(\begin{array}{ccccccc}
k_{2} & 1 & 0 & 0 & 0 & 0 & 0 \\
1 & \frac{1}{t} & 0 & 0 & 0 & 0 & 0 \\
k_{2} & 0 & 1 & 0 & 0 & 0 & 0 \\
0 & 0 & 0 & 1 & 0 & 0 & 0 \\
0 & 0 & 0 & 0 & 0 & 1 & 0 \\
k_{4} & 0 & 0 & 0 & 0 & 0 & 1 \\
0 & -1 & 0 & 0 & 1 & 0 & 0
\end{array}\right) \\
& k_{1}=-5-16 i+\sqrt{25+20 i} \text {, } \\
& k_{2}=-\frac{1}{2}(5+2 i-\sqrt{25+20 i}) \\
& k_{3}=\frac{1}{2}(3+2 i+\sqrt{25+20 i}) \\
& k_{4}=(2 i[9+2 i-\sqrt{25+20 i}+((-33-24 i)+\sqrt{565+1600 i}) t])\left(k_{1} k_{5}\right)^{-1} \\
& k_{5}=5+2 i+\sqrt{25+20 i}+2 t
\end{aligned}
$$

\section{References}

[1] Abramowitz, M. J., Clarkson, P. A. Solitons, Nonlinear Evolution Equations and Inverse Scattering. London Mathematical Society: Lecture Note Series. vol 149 Cambridge University Press (1991)

[2] Aktosun, T., Klaus, M., van der Mee, C.: Explicit Wiener-Hopf factorization for certain non-rational matrix functions. Integral Equations and Operator Theory. vol 15(6), pp. 879-900. Birkhäuser-Verlag (1992)

[3] Andrew, A. D., Green, W. L.: Spectral Theory of Operators on Hilbert Space. V School of Mathematics Georgia Institute of Technology Atlanta, GA 30332-0160 (2002)

[4] Asch, M., Lebeau, G.: The Spectrum of the Damped Wave Operator for a Bounded Domain in $R^{2}$. Experiment. Math. vol. 12(2), pp. 227-241. Euclid publishers (2003) 
[5] Ball, J.A., Clancey, K.F.: An elementary description of partial indices of rational matrix functions. Integral Equations Operator Theory. vol. 13(3), pp. 316-322 Birkhäuser-Verlag (1990)

[6] Böttcher, A., Karlovich, Yu. I.: Cauchy's Singular Integral Operator and Its Beautiful Spectrum. Oper. Theory Adv. Appl. vol. 129, pp. 109-142 Birkhäuser Basel (2001)

[7] Calderón, A.P., Cauchy integrals on Lipschitz curves and related operators. Proc. Nat. Acad. Sci. U.S.A. vol. 74(4), pp. 1324-1327 (1997)

[8] Câmara, M.C., dos Santos, A.F.: Generalised Factorization for a Class of $n \times n$ Matrix Functions - Partial Indices and Explicit Formulas. Integral Equations Operator Theory. vol. 20(2), pp. 198-230 Birkhäuser Verlag (1994)

[9] Câmara, M.C., dos Santos, A.F., Carpentier, M.: Explicit Wiewer-Hopf factorisation and non-linear Riemann-Hilbert problems. Proc. Roy. Soc. Edinburgh Sect. vol. 132(1) (A), pp. 45-74 (2002)

[10] Clancey, K., Gohberg, I.: Factorization of Matrix Functions and Singular Integral Operators. Operator Theory: Advances and Applications. vol. 3. Birkhäuser Verlag, Basel (1981)

[11] Conceição, A.C.: Factorization of Some Classes of Matrix Functions and its Applications (in portuguese). Ph.D thesis, University of Algarve, Faro (2007)

[12] Conceição, A.C.: Computing the kernel of special classes of paired singular integral operators with Mathematica software. Proceedings of the 4th International Conference on Numerical and Symbolic Computation: Developments and Applications, Ed A. Loja, J. I. Barbosa, and J. A. Rodrigues, Porto - Portugal (2019)

[13] Conceição, A.C., Kravchenko, V.G.: Factorization algorithm for some special matrix functions. Oper. Theory Adv. Appl. vol. 181, pp. 173-185 Birkhäuser Verlag (2008)

[14] Conceição, A.C., Kravchenko, V.G.: About explicit factorization of some classes of non-rational matrix functions. Math. Nachr. vol. 280(9-10), pp. 1022-1034 WILEY-VCH Verlag GmbH \& Co. KGaA, Weinheim (2007)

[15] Conceição, A.C., Kravchenko, V.G., Pereira, J.C.: Computing some classes of Cauchy type singular integrals with Mathematica software. Adv. Comput. Math. 39 (2), 273-288 (2013)

[16] Conceição, A.C., Kravchenko, V.G., Pereira, J.C.: Rational functions factorization algorithm: a symbolic computation for the scalar and matrix cases. Proceedings of the 1st National Conference on Symbolic Computation in Education and Research (CD-ROM), P02, 13 pp. Instituto Superior Tcnico, Lisboa, Portugal, April 2-3 (2012)

[17] Conceição, A.C., Kravchenko, V.G., Pereira, J.C.: Factorization Algorithm for Some Special Non-rational Matrix Functions. In Ball, J., Bolotnikov, V., Rodman, L., Helton, J., Spitkovsky, I. (eds.) Topics in Operator Theory, Operator Theory: Advances and Applications. vol. 202, pp. 87-109. Birkhäuser Verlag (2010)

[18] Conceição, A.C., Kravchenko, V.G., Teixeira, F.S.: Factorization of some classes of matrix functions and the resolvent of a Hankel operator. FSORP2003 Factorization, Singular Operators and Related Problems. Ed. S. Samko, A. Lebre, A. F. dos Santos, Funchal, Portugal, pp. 101-110. Kluwer Academic Publishers (2003)

[19] Conceição, A.C., Kravchenko, V.G., Teixeira, F.S.: Factorization of matrix funtions and the resolvents of certain operators Singular Integral Operators, Factorization and Applications - Oper. Theory Adv. Appl. vol. 142, pp. 91-100. Ed. A. Bttcher, M. A. Kaashoek, A. B. Lebre, A. F. dos Santos, F.-O. Speck, Birkhäuser Verlag (2003)

[20] Conceição, A.C., Marreiros, R.C.: On the kernel of a singular integral operator with non-Carleman shift and conjugation. Oper. Matrices. 9 (2), 433-456 (2015)

[21] Conceição, A.C., Marreiros, R.C., Pereira, J.C.: Symbolic computation applied to the study of the kernel of a singular integral operator with non-Carleman shift and conjugation. Math.Comput.Sci. vol. 10(3), pp. 365-386 (2016)

[22] Conceição, A.C., Pereira, J.C.: Using wolfram mathematica in spectral theory. Proceedings of the 3rd International Conference on Numerical and Symbolic Computation: Developments and Applications, Ed A. Loja, J. I. Barbosa, and J. A. Rodrigues, Guimarães - Portugal, pp. 295-304 (2017)

[23] Conceição, A.C., Pereira, J.C.: Exploring the spectra of some classes of singular integral operators with symbolic computation. Math.Comput.Sci. vol. 10(2), pp. 291-309 (2016) 
[24] Conceição, A.C., Pereira, J.C.: Exploring the spectra of some classes of paired singular integral operators: the scalar and matrix cases. Libertas Mathematica (new series). 34 (2), 35 pp. (2014)

[25] Davis, G.: Opérateurs intégraux singuliers sur certaines courbes du plan complexe. Ann. Sci. Ecole Norm. S. vol. 17(1), pp. 157-189 (1984)

[26] Ehrhardt, T., Speck, F.-O.: Transformation techniques towards the factorization of non-rational $2 \times 2$ matrix functions Linear Algebra Appl. vol. 353(1-3), pp. 53-90 Elsevier Science Inc (2002)

[27] Faddeev, L.D., Tkhatayan, L.A.: Hamiltonian Methods in the Theory of Solitons. Springer-Verlag (1987)

[28] Feldman, I., Gohberg, I., Krupnik, N.: An Explicit Factorization Algorithm. Integral Equations Operator Theory. vol. 49(2), pp. 149-164. Birkhäuser Verlag (2004)

[29] Feldman, I., Marcus, A.: On some properties of factorization indices Integral Equations Operator Theory. vol. 30(3), pp. 326-337. Birkhäuser Verlag (1998)

[30] Garnett, J. B.: Bounded Analytic Functions. Graduate Texts in Mathematics, vol 236. Springer, Berlin (2007)

[31] Gohberg, I., Kaashoek, M.A., Spitkovsky, I.M.: An Overview of Matrix Factorization Theory and Operator Applications. In Gohberg, I., Manojloviv, N., dos Santos, A.F. (eds.) Factorization and Integrable Systems, Operator Theory: Advances and Applications, vol. 141, pp. 1-102. Birkhäuser Verlag (2003)

[32] Gohberg, I., Krupnik, N.: The Spectrum of Singular Integral Operators in $L_{p}$ Spaces. Oper. Theory Adv. Appl. vol. 206, pp. 111-125. Birkhäuser Verlag (2010)

[33] Gohberg, I., Krupnik, N.: One-Dimensional Linear Singular Integral Equations. Operator Theory: Advances and Applications. vol. 54. Birkhäuser Verlag, Basel (1992)

[34] Gohberg, I., Krupnik, N.: One-Dimensional Linear Singular Integral Equations. Operator Theory: Advances and Applications. vol. 53. Birkhäuser Verlag, Basel (1992)

[35] Janashia, G., Lagvilava, E.: On factorization and partial indices of unitary matrix-functions of one class. Georgian Math. J. vol. 4(5), pp. 439-442. Kluwer Academic Publishers-Plenum Publishers (1997)

[36] Kravchenko, V.G. , Lebre, A.B., Litvinchuk, G.S.: Spectrum problems for singular integral operators with Carleman shift. Math. Nachr. 226, 129-151 (2001)

[37] Kravchenko, V.G., Lebre, A.B., Rodriguez, J.S.: Factorization of singular integral operators with a Carleman shift and spectral problems. J. Integral Equations Appl. 13 (4), 339-383 (2001)

[38] Kravchenko, V.G., Litvinchuk, G.S.: Introduction to the Theory of Singular Integral Operators with Shift. Mathematics and its Applications. vol. 289. Kluwer Academic Publishers, Dordrecht (1994)

[39] Kravchenko, V.G., Migdal'skii, A. I.: A regularization algorithm for some boundary-value problems of linear conjugation. Dokl. Math. vol. 52, pp. 319-321 (1995)

[40] Kravchenko, V.G., Nikolaichuk, A.M.: On partial indices of the Riemann problem for two pair of functions. Soviet Math. Dokl. vol. 15 , pp. 438-442 (1974)

[41] Litvinchuk, G.S.: Solvability Theory of Boundary Value Problems and Singular Integral Equations with Shift. Mathematics and its Applications, vol. 523. Kluwer Academic Publishers, Dordrecht (2000)

[42] Litvinchuk, G.S., Spitkovskii, I.M.: Factorization of Measurable Matrix Functions. Operator Theory: Advances and Applications, vol. 25. Birkhäuser Verlag, Basel (1987)

[43] Mikhlin, S.G., Prössdorf, S.: Singular Integral Operators. Springer- Verlag (1986)

[44] N. I. Muskhelishvili, Singular Integral Equations, Dover Publications, 1992.

[45] Nikol'skii, N. K.: Treatise on the Shift Operator. Spectral Function Theory. Grundlehren der mathematischen Wissenschaften, vol. 273, Springer-Verlag Berlin (1986)

[46] Plemelj, J.: Riemannshe Funktionenscharen mit gegebener Monodromiegruppe. Monat. Math. Phys. vol. 19, pp. 211-245 (1908)

[47] S. Prössdorf, Some Classes of Singular Equations, North-Holland, 1978.

[48] Rudin, W.: Real and Complex Analysis. McGraw-Hill (1987)

[49] Voronin, A.F.: A method for determining the partial indices of symmetric matrix functions. Sib. Math. J. vol. 52, pp. 41-53 (2011) 
Ana C. Conceição

Center for Functional Analysis, Linear Structures and Applications (CEAFEL)

Departamento de Matemática, Universidade do Algarve, 8005-139 Faro, Portugal

+351962812612

e-mail: aconcei@ualg.com

orcid: 0000-0001-7103-3588 